\title{
Anton Wilhelm Amo oder eine verpasste Chance äquipollenter Integration
}

Wechseln wir an dieser Stelle von der frühen Entwicklung der Aufklärung in Frankreich zur Situation der Frühaufklärung in Deutschland. Wir verlassen damit nur scheinbar den Bereich jener Sprachen, die sich vom Lateinischen herleiten. Zwischen Gottfried Wilhelm Leibniz und Immanuel Kant nahm der 1679 in Breslau geborene und 1754 in Halle verstorbene Christian Wolff die sicherlich herausgehobenste Stellung ein. Wolff ging in seinen philosophischen Schriften vom Lateinischen, der damals im deutschen Sprachraum noch vorherrschenden Wissenschaftssprache, zur deutschen Sprache über, deren Möglichkeiten er grundlegend erweiterte. Angefügt sei, dass ihm bei seinem reichen Schaffen eine Vielzahl deutschsprachiger Begrifflichkeiten ebenso in der Philosophie wie in der Rechtswissenschaft oder der Theologie bis heute $\mathrm{zu}$ verdanken sind.

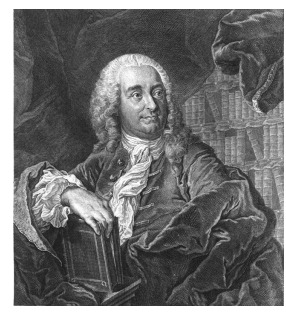

Abb. 18: Johann Martin Bernigeroth: Christian Wolff (1679-1754).

Nachdem er im Jahr 1706 Professor für Philosophie und Mathematik an der Universität Halle geworden war, stieg er zu einem der international berühmtesten deutschen Philosophen der Aufklärung auf, insofern er wie nach ihm Montesquieu 1710 als Mitglied in die Royal Society und im Folgejahr in die Berliner Akademie der Wissenschaften aufgenommen wurde. Hohe akademische Mitgliedschaften in Frankreich und Russland folgten. Christian Wolff war zweifellos die herausragende Gestalt der Hallenser Universität. Er behielt diese Stellung bis er sich mit Schriften der chinesischen Philosophie beziehungsweise den lateinischen Übersetzungen des Konfuzius wie des Menzius auseinandersetzte und in der Folge 1721, im Erscheinungsjahr von Montesquieus Lettres persanes, seine berühmte Rede über die praktische Philosophie der Chinesen hielt. In ihr unterstrich er die Existenz einer Ethik, die sich unabhängig vom christlichen Glauben im Kulturbereich Chinas entwickelt habe. Wolff stand mit seinen Ansichten für ein gegenüber kulturellen Differenzen offenes Denken ein, das ihn als einen der wesentlichen deutschen Aufklärer auszeichnete. Auch während der folgenden Jahrzehnte erwies 
sich Wolff als entscheidende Brücke zwischen europäischem Denken und chinesischen Traditionen, mit denen er sich ein Leben lang beschäftigte.

Doch eine solche Rede genügte in Preußen 1721, um von der in Halle stark vertretenen Fraktion der Pietisten persönlich angegriffen und des Atheismus beschuldigt zu werden. 1723 musste Christian Wolff auf Grund der Auslegungen seiner Hallenser Rede sein Amt aufgeben und auf Geheiß des preußischen Königs Friedrich Wilhelm I. binnen achtundvierzig Stunden Halle verlassen. Die Gegner der Aufklärung hatten zugeschlagen - nicht zum letzten Mal in Halle. Es war eine Verbannung, die erst 1740, gleich zu Beginn der Amtszeit Friedrichs II. von Preußen, wieder aufgehoben wurde, als der junge aufgeklärte Monarch Christian Wolff wieder nach Halle zurückholte. Diese Auseinandersetzungen mögen uns einen Vorgeschmack auf jene große, aber bislang vernachlässigte Figur in der Geschichte der Philosophie in Deutschland sowie der deutschen Philosophie namens Anton Wilhelm Amo geben, mit der wir uns in diesem Kapitel unserer Vorlesung beschäftigen wollen. Denn Amo war als junger Student ein glühender Anhänger jenes Christian Wolff, der aus Halle vertrieben worden war, dessen tolerante Traditionen aber nachwirkten.

Wie, Sie haben den Namen Anton Wilhelm Amo noch nie gehört? Dann darf ich Ihnen verraten, dass seit kurzem im Herzen Berlins eine Straße, die ehemalige Mohrenstraße, nach diesem deutschen Philosophen benannt ist. Doch wer war dieser Mann? ${ }^{1}$ Seine Schriften erschienen, gegenläufig zu Wolff, in lateinischer Sprache, sind aber heute noch immer nicht einfach $\mathrm{zu}$ beziehen und konsultierbar. Die Amo-Forschung steckt noch in den Kinderschuhen. Denn die deutsche Philosophie oder die Philosophie in Deutschland hat sich bislang wenig - und dies ist ein Euphemismus - um diesen Aufklärungsphilosophen gekümmert und bemüht. Er gehört zweifellos zu den verschütteten Traditionen unserer Geschichte, welche ich gerne mit Ihnen gemeinsam freilegen will.

Nun, an einem 9. Juni des Jahres 1727 trug sich ein junger Mann unter der laufenden Nummer 488 in die Matrikel der damals preußischen Universität Halle ein, ein Mann, der Philosophiegeschichte schrieb, ohne doch bislang in die deutsche Philosophiegeschichte wirklich als feste Größe eingegangen zu sein. Die eigenhändige Eintragung ${ }^{2}$ hält fest, dass der neue Student an der erst 1694, also wenige

1 Vgl. hierzu die handliche Monographie von Ette, Ottmar: Anton Wilhelm Amo: Philosophieren ohne festen Wohnsitz. Eine Philosophie der Aufklärung zwischen Europa und Afrika. Zweite Auflage mit neuem Vorwort. Berlin: Kulturverlag Kadmos 2020.

2 Vgl. Abb. 7 in Brentjes, Burchard: Anton Wilhelm Amo. Der schwarze Philosoph in Halle. Leipzig: Koehler \& Amelang 1976, S. 32/33. 
Jahrzehnte zuvor gegründeten Universität ${ }^{3}$ aus Afrika, „aus Axim in Guinea“, stammte und den Namen Anton Wilhelm Amo trug. Wer war dieser junge Mann?

Anton Wilhelm Amo kam nicht in Halle, Deutschland oder Europa zur Welt, sondern in Afrika. Mit dieser Einschreibung nahm Amo „als erster und für über 220 Jahre zugleich letzter Mensch afrikanischer Herkunft das Studium der Philosophie und Rechtswissenschaft an der Universität Halle auf“" ${ }^{4}$ Amo war in jeglicher Hinsicht ein Einzelfall, in vielerlei Hinsicht aber auch charakteristisch für ein Jahrhundert, das mit all seinen Widersprüchen ein treffliches Experimentierfeld für künftige Entwicklungen abgab. Von der Zahlung der Immatrikulationsgebühren war er offenkundig auf Veranlassung seiner Förderer, von denen wir gleich sprechen werden, befreit worden. Mit dieser ordentlichen Einschreibung begann eine akademische Karriere, von welcher der international berühmte, an der Universität Göttingen lehrende Anthropologe und Schädelkundler Johann Friedrich Blumenbach noch im Jahre 1790, mithin aus großer zeitlicher Distanz, aber offenkundig wohlinformiert festhielt:

Unser seel. Hollmann hat, da er noch Prof. in Wittenberg war, a. 1734 den Neger Ant. Wilh. Amo zum D. der Weltweisheit creirt, der sich sowohl in Schriften als auch als Docent vortheilhaft gezeigt hat, und von welchem ich zwey Abhandlungen vor mir habe, wovon zumal die eine viele unerwartete und wohlverdaute Belesenheit in den besten physiologischen Werken jener Zeit verrätht. In einer Nachricht von Amo's Leben, die bey dieser Gelegenheit im Namen des academischen Concilii gedruckt worden, wird seiner ausnehmenden Rechtschaffenheit, so wie seiner Fähigkeiten, seinem Fleiss und seiner Gelehrsamkeit grosses Lob ertheilt. Es heisst z. B. von seinen philosophischen Vorlesungen excussis tam veterum quam novorum placitis, optima quaeque selegit, selecta enucleate ac dilucide interpretatus est u.s.w. ${ }^{5}$

Johann Friedrich Blumenbach war der große deutsche Spezialist für außereuropäische Wissenschaften und einer der Lehrmeister Alexander von Humboldts an der Universität Göttingen, die als universitäre Neugründung des 18. Jahrhunderts rasch zu einer der großen Bildungseinrichtungen im deutschen Sprachraum geworden war. Der angeführte Exkurs des großen Blumenbach in seinen Beyträgen zur Naturgeschichte auf einen „Neger“ namens Anton Wilhelm Amo drückt das

3 Vgl. Abraham, William E.: The Life and Times of Anton Wilhelm Amo, the First African (Black) Philosopher in Europe. In: Asante, Molefi Kete / Abary, Abu S. (Hg.): African Intellectual Heritage. A Book of Sources. Philadelphia: Temple University Press 1996, S. 429.

4 Arndt, Susan: Die 100 wichtigsten Fragen: Rassismus. München: Verlag C.H. Beck 2012, S. 143. Vgl. auch bzgl. der Geschichte des Rassismus in Deutschland Hund, Wulf D.: Wie die Deutschen weiß wurden. Kleine (Heimat)Geschichte des Rassismus. Stuttgart: Metzler 2017.

5 Blumenbach, Johann Friedrich: Beyträge zur Naturgeschichte. 2 Bände. Erster Theil. Göttingen: Johann Christian Dieterich 1790, S. $98 \mathrm{f}$. 
Erstaunen, aber auch die Anerkennung jenes Mannes aus, der nicht nur als einer der einflussreichsten wissenschaftlichen Lehrer eines Georg Forster und vieler weiterer Reisender im späteren18. Jahrhundert gewirkt hatte, sondern auch als einer der maßgeblichen Erfinder von Rassenkategorien und Rassenhierarchien gelten muss. ${ }^{6}$ Die Anerkennung des für seine schwarze Haut weithin bekannten Philosophen, ${ }^{7}$ der - wie Blumenbach an anderer Stelle ausführte - in der Folge als „Hofrat $^{\text {“8 }}$ an den Preußischen Hof nach Berlin gegangen sei, gerade aus dem Munde eines das Rassendenken so befördernden Wissenschaftlers mag erstaunen; und doch blieb der Name Anton Wilhelm Amo dem 19. Jahrhundert wohl in weiten Kreisen weitgehend unbekannt. Ich hoffe, dass sich dies im 21. Jahrhundert grundlegend ändern wird, muss aber gestehen, dass meine eigenen Recherchen zum von Blumenbach erwähnten Aufenthalt Amos in Berlin und am preußischen Hof bislang noch keine Früchte getragen haben.

Doch gewiss: Auch das Schweigen rund um diesen Anton Wilhelm Amo war und ist beredt. Denn längst ist es an der Zeit, die ebenso für seine Epoche charakteristischen wie für die Zukunft wegweisenden Dimensionen seines Lebens und Denkens in einem möglichst weiten Umfeld jenseits allen Spezialistentums wahrzunehmen und ins öffentliche Bewusstsein zu heben. Es hat mir stets gefallen, mir den Weltweisen Amo in Sanssouci vorzustellen. Und ich bin sicher, dass sich eines Tages in den preußischen Staatsarchiven Spuren von Amo am preußischen Königshof von Friedrichs dem Großen - mit dem wir uns noch beschäftigen werden - finden lassen werden.

Nicht allein die Lettres persanes von Montesquieu, sondern auch die faszinierende Gestalt Anton Wilhelm Amos führt uns vor Augen, mit welcher Komplexität die so widersprüchliche Zeit der Frühaufklärung zu betrachten und inwieweit es längst überfällig ist, die Aufklärung in ihrer Gesamtheit als transareales, ${ }^{9}$ unterschiedlichste kulturelle Areas miteinander verbindendes weltweites Netzwerk zu

6 Vgl. hierzu Painter, Nell Irvin: The History of White People. New York - London: W.W. Norton 2010, S. 72-90.

7 Vgl. diese Formulierung im Aufsatz von Damis, Christine: Le philosophe connu pour sa peau noire: Anton Wilhelm Amo. In: Rue Descartes: Collège international de philosophie (Paris) (juin 2002), S. 115-127.

8 Blumenbach, Johann Friedrich: Von den Negern. Einige naturhistorische Bemerkungen bey Gelegenheit einer Schweizerreise. In: Magazin für das Neueste aus der Physik und Naturgeschichte (Gotha) IV, 3 (1787), S. 9; vgl. hierzu Lochner, Norbert: Anton Wilhelm Amo. A Ghana Scholar in Eighteenth Century Germany. In: Transactions of the Historical Society of Ghana (Achimota) III, 3 (1958), S. 178.

9 Vgl. zu diesem Begriff und der auch in der vorliegenden Studie zugrunde gelegten Globalisierungsgeschichte Ette, Ottmar: TransArea. Eine literarische Globalisierungsgeschichte. Berlin - Boston: Walter de Gruyter 2012. 
begreifen. Der ,schwarze Philosoph“ in Deutschland hat es, wie wir noch sehen werden, durchaus zu internationaler Bekanntheit gebracht. Der Hinweis auf einen namenlos bleibenden Philosophen aus Guinea in Francisco Javier Clavijeros Alter Geschichte Mexicos warf hierauf ein scharfes Licht. Denn der neuspanische Philosoph, der zweifellos von Amo gehört hatte, formulierte seine Sichtweise einer möglichst weltumspannenden und auch auf Europa zurückstrahlenden Aufklärung wie folgt:

Wollte ein Philosoph aus Guinea ein Werk unternehmen, das sich am Modell von Cornelius de Pauw ausrichtete und den Titel Philosophische Untersuchungen über die Europäer trüge, so könnte er sich desselben Argumentes wie de Pauw bedienen, um etwa die Schädlichkeit des Klimas von Europa und die Vorzüge des Klimas von Afrika zu beweisen. ${ }^{10}$

Doch greifen wir der Debatte um die Neue Welt und damit einer weltweit geführten Diskussion über die Frage, was Aufklärung ist und von wo aus warum über wen aufgeklärt wird, nicht vor. Es steht jedenfalls zu vermuten, dass der neuspanische, im späteren Mexiko aufgewachsene Philosoph Francisco Javier Clavijero, der sehr wohl die Schriften des Leo Africanus kannte, in seiner erstmals 1780 in vier Bänden erschienenen Alten Geschichte Mexicos von der Existenz Anton Wilhelm Amos wusste oder zumindest gehört hatte. Schriftliche Bezugsquellen für ein solches Wissen gab es in der internationalen République des Lettres damals reichlich. Anton Wilhelm Amo wurde so aus einer neuspanischen und damit amerikanischen Perspektive beispielhaft zur Partei in einer Debatte, die - wie wir noch sehen werden - von der Hauptstadt Preußens ausging, weltweite Dimensionen erreichte, aber in Preußen und Deutschland heute weitestgehend vergessen ist. Das sorgsame Verschütten dieser Traditionen sagt uns freilich viel über unsere Geschichte und auch viel über die gegenwärtigen zeitgeschichtlichen Kontexte, welche eine Revision dieses Verschüttens heute angehen oder ihr aber entgegenwirken. Die kleine, aber zum Teil hitzig geführte Berliner Debatte um die Umbenennung der Mohrenstraße ist gleichsam eine winzige Mise en abyme jener Debatten, denen wir uns mit Blick auf das 18. Jahrhundert nähern.

Gute Gründe sprechen folglich dafür, sich den bedeutungsvollen Namen dieses jungen Studenten an der Universität von Halle an der Saale einzuprägen, zumal er sich aus sehr verschiedenen Bestandteilen zusammensetzt, die uns im Grunde einen schon in sich differenzierten Zugang zu dieser ebenso für die afrikanische wie die europäische Ideengeschichte der Aufklärung wichtigen Persön-

10 Clavijero, Francisco Javier: Historia Antigua de México. Prólogo de Mariano Cuevas. Edición del original escrito en castellano por el autor. México: Editorial Porrúa ${ }^{7} 1982$, S. 462. 
lichkeit erlauben. ${ }^{11}$ Die lange Rezeptionsgeschichte dieses Philosophen und Rechtswissenschaftlers vermochte sich nicht nur in Deutschland, sondern auch international nur höchst schleppend zu entwickeln. Der Lebensweg wie die philosophische Entfaltung des Anton Wilhelm Amo beleuchten dennoch aus heutiger Sicht Aspekte, wie sie für ein Verständnis der nicht nur europäischen Aufklärung wie auch des philosophischen Diskurses der (ebenfalls nicht allein europäischen) Moderne $^{12}$ von großer Bedeutung sind. Denn Anton Wilhelm Amo entwickelte sich im Verlauf seines Lebens in der ersten Hälfte des 18. Jahrhunderts zweifellos zu einem für die Aufklärung charakteristischen Denker.

Doch versuchen wir, die Erwartungen nicht zu hoch zu schrauben! Anton Wilhelm Amo war sicherlich keine Schlüsselfigur für das Denken der Aufklärung; und doch vermag er uns einen wichtigen Schlüssel für ein anderes, adäquateres Verständnis von Aufklärung und Moderne an die Hand zu geben. Denn in diesem deportierten Sklaven und verehrten Doktor der Philosophie, in diesem ,Versuchsobjekt‘ gnädiger Fürsten und selbstbewusster Denker der Frühaufklärung in Preußen blitzt etwas auf von dem, was wir als eine verschüttete Geschichte Europas bezeichnen könnten, was also in der Geschichte nicht mehr präsent zu sein scheint und doch nicht aufhören kann zu sein. Denn die Geschichte Europas ist auch in der ersten Hälfte des Jahrhunderts der Aufklärung und damit noch vor der Berliner Debatte um die Neue Welt ${ }^{13}$ voller Widersprüche und enthält nicht nur die fiktionale Einbeziehung anderer ,aufklärerischer' Blickpunkte wie etwa in den Lettres persanes, sondern auch Zeugnisse und Dokumente, welche die Präsenz außereuropäischen Denkens in Europa belegen. Im Zentrum dieser Diskussionen stand die Frage, wie wir in Differenz und Frieden zusammenleben wollen und wie sich ein in sich selbst zerstrittenes und doch immer ähnliche Interessen verfolgendes Europa im weltweiten Kontext definieren kann und soll. Denn all diese Fragen berührt das Leben wie das Schreiben des jungen Mannes aus Afrika.

11 Zur Problematik des Nachnamens Amo vgl. u. a. Mabe, Jacob Emmanuel: Anton Wilhelm Amo interkulturell gelesen. Nordhausen: Verlag Traugott Bautz 2007, S. 16 f. Die oftmals spekulativen Dimensionen vieler Recherchen zum Nachnamen des verschleppten Jungen sind in der Amo-Literatur kaum zu übersehen.

12 Vgl. hierzu Habermas, Jürgen: Der philosophische Diskurs der Moderne. Zwölf Vorlesungen. Frankfurt am Main: Suhrkamp 1985.

13 Vgl. Ette, Ottmar: Von Rousseau und Diderot zu Pernety und de Pauw: Die Berliner Debatte um die Neue Welt. In: Dill, Hans-Otto (Hg.): Jean-Jacques Rousseau zwischen Aufklärung und Moderne. Akten der Rousseau-Konferenz der Leibniz-Sozietät der Wissenschaften zu Berlin am 13. Dezember 2012 anlässlich seines 300. Geburtstages am 28. Juni 2012 im Rathaus BerlinMitte. Berlin: Leibniz-Sozietät der Wissenschaften (= Sitzungsberichte der Leibniz-Sozietät der Wissenschaften 117) 2013, S. 111-130. 
Wie aber darf man sich die Geschichte Anton Wilhelm Amos vorstellen? Etwa zwanzig Jahre vor seiner Immatrikulation, am 29. Juli 1707, war der Knabe in der Schlosskapelle Salzthal zu Wolfenbüttel evangelisch getauft worden, wodurch sich erstmals ein schriftliches Zeugnis seines von den scharfen Widersprüchen seiner Zeit zutiefst geprägten Lebens findet. Denn der um die Wende zum 18. Jahrhundert im heutigen Ghana geborene, wohl zu den westafrikanischen Nzema und damit zur ethnischen Gruppe der Akan gehörende und zunächst in seiner Heimat aufgewachsene Junge war seinen Eltern entrissen und versklavt worden. In der Folge wurde er wohl im Jahr 1704 von der holländischen WestIndischen Kompanie nach Amsterdam verbracht, ein Weg, der ihn mit großer Wahrscheinlichkeit an Bord eines holländischen Sklaven- oder Handelsschiffes zunächst in die Karibik (wo sein Bruder später als Sklave in der Kolonie Surinam arbeiten musste) und von dort aus dann in den Westen Europas führte. Ein ungewöhnlicher Weg? Nicht für die Unzahl an Opfern des europäischen „Slave Trade“ über den Black Atlantic. ${ }^{14}$

Als eine Art Werbe-Geschenk ${ }^{15}$ der Holländer Händler ging der Junge in das Eigentum eines kunst- und feinsinnigen Herzogs über. Dass die Vertreter der so mächtigen holländischen Handelsgesellschaft dies tun konnten, weist unzweifelhaft darauf hin, dass es sich um einen Sklaven handelte, über den man selbstverständlich frei verfügen zu können glaubte. In der Tat gibt es - wie bereits erwähnt - Zeugnisse und Indizien, die dafür sprechen, dass zumindest ein Bruder des Knaben als Sklave nach Surinam ${ }^{16}$ verschleppt und damit in das Sklaverei-System der holländischen Karibik, der holländischen „West Indies“, verbracht wurde.

Vieles deutet im Übrigen darauf hin, dass die Herzöge von BraunschweigWolfenbüttel auf vielfache Weise in den transatlantischen Sklavenhandel verstrickt waren. ${ }^{17}$ Eine Ausnahme bildeten sie diesbezüglich weder auf deutscher noch auf europäischer Ebene. Mit der physischen Überführung des jungen Westafrikaners von der im europäischen beziehungsweise holländischen Kolonialsys-

14 Vgl. hierzu die längst klassische Studie von Gilroy, Paul: The Black Atlantic. Modernity and Double Consciousness. London: Verso 1993; sowie den Band Der Black Atlantic. Herausgegeben vom Haus der Kulturen der Welt in Zusammenarbeit mit Tina Campt und Paul Gilroy. Berlin: Haus der Kulturen der Welt 2004.

15 Vgl. hierzu Abraham, William E.: Anton Wilhelm Amo. In: Wiredu, Kwasi (Hg.): A Companion to African Philosophy. Advisory Editors William E. Abraham, Abiola Irele and Ifeanyi A. Menkiti. Blackwell Publishing 2004, S. 191; Vorbehalte gegen diese dominante Annahme formulierte Mabe, Jacob Emmanuel: Anton Wilhelm Amo interkulturell gelesen, S. 13.

16 Vgl. zur Einschätzung dieses Faktums auch Brentjes, Burchard: Anton Wilhelm Amo. Der schwarze Philosoph in Halle, S. 30. Verwiesen sei hier auch auf das Zeugnis des Schweizer Schiffsarztes Gallandat, auf das noch einzugehen sein wird.

17 Vgl. hierzu Mabe, Jacob Emmanuel: Anton Wilhelm Amo interkulturell gelesen, S. $14 \mathrm{f}$. 
tem verankerten Institution Sklaverei ${ }^{18}$ in das Herzogtum Braunschweig-Wolfenbüttel, in dem nominell keine Sklaverei bestand, war letztlich eine Fortführung dieses versklavten Zustands außerhalb dieser Institution verbunden.

War damit Amos Sklaven-Status ein Ende gesetzt? Keinesfalls! So spricht Michael Zeuske mit Blick auf Anton Wilhelm Amo wie auch auf viele andere historische Figuren mit guten Gründen davon, dass es sich hier um „versklavte Schwarze in Europa“ im Rahmen einer „Eigentums-Sklaverei“ gehandelt habe ${ }^{19}$. Amo war in das Eigentum einer anderen Person übergegangen: Es gab in Europa Sklaven ohne Sklaverei, ${ }^{20}$ eine Feststellung, die auf den ersten Blick nicht zum europäischen Jahrhundert der Aufklärung passen will. Und doch gehört dies zu den Widersprüchen der europäischen Aufklärung: Nicht wenige der - auch in unserer Vorlesung behandelten - Aufklärer waren in den transatlantischen Sklavenhandel verstrickt.

Anton Ulrich, der für sein engagiertes Eintreten für die Künste und Wissenschaften wie auch als Verfasser zweier Romane bekannte regierende Herzog von Braunschweig-Wolfenbüttel, nahm das ihm von der West-Indischen Kompanie überbrachte ,Geschenk' an und verlieh zusammen mit seinem nicht weniger der Aufklärung zuneigenden Sohn Wilhelm August dem schwarzen Jungen die fürstlichen Vornamen als Taufnamen. Die bereits erwähnte Taufe des Jahres 1707, die in die Zeit vor den Übertritt des Herzogs im Jahre 1709 zum katholischen Glauben fiel, widerspricht im Übrigen der von William E. Abraham vorgetragenen These, ${ }^{21}$ dass der Junge aus einer bereits christlichen (und damit notwendig getauften) Familie aus einem Dorf bei Axim im heutigen Ghana ${ }^{22}$ stammen könnte. Sie führt vielmehr im Zeichen des christlichen Glaubens den wahrscheinlich, wenn auch nicht unumstritten, von der damaligen Goldküste stammenden Namen $\mathrm{Amo}^{23}$ mit

18 Vgl. hierzu den einführenden Essay „Sklavereien statt Sklaverei: Ein historisch-anthropologischer Essay“ in Zeuske, Michael: Handbuch Geschichte der Sklaverei. Eine Globalgeschichte von den Anfängen bis zur Gegenwart. Berlin - Boston: Walter de Gruyter 2013, S. 1-26.

19 Ebda., S. 517; vgl. auch Arndt, Susan: Die 100 wichtigsten Fragen: Rassismus, S. $142 \mathrm{f}$.

20 Vgl. Zeuske, Michael: Handbuch Geschichte der Sklaverei. Eine Globalgeschichte von den Anfängen bis zur Gegenwart. Berlin - Boston: Walter de Gruyter 2013, S. 517.

21 Vgl. Abraham, William E.: The Life and Times of Anton Wilhelm Amo, the First African (Black) Philosopher in Europe. In: Current Anthropology (Chicago) XVI, 3 (September 1975), S. 426.

22 Vgl. Brentjes, Burchard: Anton Wilhelm Amo, First African Philosopher in European Universities, S. 443.

23 Schon Wolfram Suchier mutmaßte, es könne sich um einen Familiennamen von der Goldküste handeln, aber auch um eine Benennung durch die christliche Liebe, wobei der kleine ,Mohr` am Hofe von Anton Ulrich aber auch bisweilen den Amor dargestellt haben könnte; vgl. Suchier, Wolfram: A.W. Amo. Ein Mohr als Student und Privatdozent der Philosophie in Halle, Wittenberg und Jena 1727 / 1740. In: Akademische Rundschau. Zeitschrift für das gesamte 
den Vornamen eines europäischen Fürstenhauses zusammen, das sich ganz ,natürlich‘ im Besitz afrikanischer Diener befand. Denn in dieser Hinsicht war ,der Fall Amo‘ in der Tat nicht außergewöhnlich.

So wurde der kleine Anton Wilhelm Amo zu einem Teil jener schwarzen Dienerschaft, die man sich wie auch an vielen anderen Fürstenhöfen als exotistisches Statussymbol und Zeichen eigener Machtfülle zur Verfügung wie zum Vergnügen ,hielt‘. Sie glauben mir das nur schwer? Dann machen Sie mal einige Schritte von der Universität in den Park Sanssouci, wo sie am östlichen Ende auf das sogenannte „Mohren-Rondell“ stoßen werden! Selbst die Touristenführer in Schloss und Park wissen bisweilen über diese Seite der Geschichte nichts.

Damit aber entwickelte sich Amos Leben, der seit Ende seiner Studienjahre seinem Nachnamen die Bezeichnung „Afer“ beziehungsweise „Guinea Afer“ hinzuzufügen begann, von Beginn an in einem Spannungsfeld, das in geographischer Hinsicht von Westafrika und der Karibik, Holland und Deutschland gebildet wurde. Es handelt sich um eine Tatsache, die schon auf dieser Ebene die spezifisch transareale Dimension des Lebens, aber auch Schaffens Anton Wilhelm Amos unübersehbar vor Augen führt. Es gibt noch viele Zeichen dieser Geschichte aus jener nicht allzu fernen Zeit wie etwa auch die Bezeichnung „Mohrenstraße“ oder „Mohren-Apotheke“: Wir müssen sie nur zu verstehen lernen ...

Räumen wir an dieser Stelle ein: Die Herzöge von Braunschweig-Wolfenbüttel ließen ihrem Patenkind zweifellos eine überdurchschnittlich gute Behandlung zuteilwerden, zumindest wenn wir diese mit den Lebensbedingungen anderer schwarzer Diener und Pagen an europäischen Fürstenhöfen vergleichen. Wäre dies nicht der Fall gewesen, wir hätten niemals mehr etwas von diesem ,Sklaven ohne Sklaverei` gehört. Damit soll keineswegs das ungeheure, vom Rassedenken stark geprägte Spannungsverhältnis geleugnet oder auch nur relativiert werden, mit dem sich Anton Wilhelm Amo von Beginn seines Lebens an konfrontiert sah. Er wurde für seine Herren, für seine ,Besitzer` zum Experiment, zum Versuchsobjekt, an dem die Bildungsfähigkeit ,des` Afrikaners erprobt werden sollte; und er verwandelte sich durch die Nutzung dieser Chance vom Objekt einer an ihm vollzogenen Handlung in ein handelndes Subjekt, in den ,Herren“ (Span. „amo“) seines Schicksals, das ihn freilich niemals gänzlich frei zur Entfaltung kommen ließ.

Nur wenig wissen wir über die Zeit zwischen Anton Wilhelm Amos Taufe und seiner Einschreibung an der aufstrebenden preußischen Universität Halle,

Hochschulwesen und die akademischen Berufsstände (Leipzig) IV, 9-10 (Juni - Juli 1916), S. 441-448, hier, S. 443. 
die zum damaligen Zeitpunkt für ihre herausragende Rolle innerhalb der deutschen Frühaufklärung bekannt war. Noch immer wirkte in Halle der Ruf Wolffs nach, aller Pietisten zum Trotz. Doch einige Belege gibt es aus dieser voruniversitären Zeit, die für Amos geistige Entwicklung sicherlich wichtig, doch für seine intellektuelle Statur zweifellos nicht entscheidend waren. Kammerrechnungen des Wolfenbüttelers Hofs aus den Jahren 1716 und 1717 sowie 1720 und 1721 sowie eine handschriftliche Quittung Amos über erhaltenes Kostgeld belegen wiederholte Zahlungen an den jungen Mann und lassen vermuten, dass er für Dienste bei Hofe entlohnt wurde. Doch bleibt bis heute noch immer unklar, welche Ausbildung Anton Wilhelm Amo in Wolfenbüttel genoss, ob er die in vielerlei Hinsicht ausgezeichnete Ritter-Akademie von Wolfenbüttel besuchen durfte ${ }^{24}$ - Wolfram Suchier brachte auch das berühmte Hallische Waisenhaus ins Spiel ${ }^{25}$ - oder sich gar zunächst zwischen 1721 und 1727 an der Landesuniversität des Herzogtums in Helmstedt immatrikulierte. ${ }^{26}$ Die Amo-Forschung wird diese Lücken sicherlich künftig schließen; doch für die Ausrichtung unserer Überlegungen ist dies nicht von entscheidender Relevanz.

Keine dieser Hypothesen konnte jedenfalls bis heute schlüssig belegt werden. Erblickten seine Herren in ihm noch immer den Sklaven, der ihnen zum Geschenk gemacht worden war, sahen sie in ihm ein Versuchsobjekt, mit dem man Experimente veranstalten konnte, oder die Chance, eine (im europäischen Sinne verstandene) humanistische Ausbildung im Geiste der Frühaufklärung auch Bewohnern anderer Weltteile zukommen zu lassen? Wie auch immer man diese Fragen beantworten mag: Anton Wilhelm Amo wurde zu einem Studenten in Preußen. Und er wuchs zu einem jungen Intellektuellen heran, der seinen eigenen Kopf hatte und seinen eigenen Weg in der Frühaufklärung ging.

Als Anton Wilhelm Amo nach Halle und damit in eines der Zentren der deutschen Frühaufklärung kam, hatten dort - wie bereits angedeutet - die Auseinandersetzungen zwischen Pietisten und aufklärerischen Rationalisten ihren ersten großen Höhepunkt überschritten. Die Verbannung des längst international renommierten Aufklärungsphilosophen Christian Wolff durch den Preußischen König war längst ein Faktum, und dass dies auf Betreiben der Klerikalen erfolgt war ebenso. Anton Wilhelm Amo galt als junger Student wie später als Universitätsdozent lange Zeit als glühender Verehrer Wolffs. Doch auch wenn Carl Günter Ludo-

24 Vgl. Abraham, William E.: The Life and Times of Anton Wilhelm Amo, the First African (Black) Philosopher in Europe, S. 429.

25 Vgl. Suchier, Wolfram: A.W. Amo, S. 443.

26 Vgl. Ebda., S. 444; Lochner, Norbert: Anton Wilhelm Amo. A Ghana Scholar in Eighteenth Century Germany, S. 170f; sowie (ders.): Anton Wilhelm Amo. In: Übersee-Rundschau (Hamburg) 10 (1958, S. 22-31, und Arndt, Susan: Die 100 wichtigsten Fragen: Rassismus, S. 143. 
vici, selbst ein vehementer Vertreter Wolffs, seinen Zeitgenossen Amo auch als „einen fürnehmlichen Wolffianer“ ${ }^{27}$ bezeichnete - eine Verortung, die bis heute höchst umstritten ist ${ }^{28}$-, so kann der in Afrika geborene Philosoph zumindest in sprachlicher Hinsicht kaum als ein solcher gelten, da er selbst in seiner Sprachenwahl anders vorging.

Denn zählte Christian Wolff zu jenen Philosophen der deutschen Frühaufklärung, die sich maßgeblich und nachhaltig für die Entfaltung des Deutschen als Sprache der Philosophie in Wort und Tat einsetzten, so wählte Anton Wilhelm Amo doch von Beginn an, aber auch in seiner späteren wissenschaftlichen Entwicklung die lateinische Sprache als Idiom seiner akademischen Kommunikation. Ein eher ,konservativer‘, rückschrittlicher Akt? Oder gar ein stilles Einverständnis mit den orthodoxen Kräften an der vom preußischen Herrscher gemaßregelten Universität?

Keineswegs: Aus heutiger Perspektive erscheint die Sprachenwahl Amos als konsequent! Denn für die langfristige Entscheidung zugunsten des Lateinischen dürften gute Gründe den Ausschlag gegeben haben. Zum einen war Latein an deutschen Universitäten noch immer die (wenn auch keineswegs mehr unbestritten) dominante Sprache der Wissenschaft, verfügte es gerade in Deutschland doch noch immer über ein hohes Prestige als international verständliche Gelehrtensprache, in der man sich über die Landesgrenzen hinweg verständigen konnte. Zum anderen befand sich das Deutsche im Bereich der Philosophie zweifellos noch in den Kinderschuhen, folglich in einer Phase der Konstituierung und begrifflichen Ausdifferenzierung, deren Ergebnis noch offen war. Innerhalb einer französischsprachigen République des Lettres sollte es auch künftig eine nur marginale Rolle spielen. Das Lateinische aber war - und dies dürfte für Amo das entscheidende Element seiner Sprachenwahl gewesen sein - bereits zu Beginn der ersten Phase beschleunigter Globalisierung neben dem Spanischen und Portugiesischen zu einer globalisierten Weltsprache geworden, in der grundlegend wichtige Schriften nicht allein in Europa, sondern gerade auch in den außereuropäischen Kolonien insbesondere Amerikas verfasst und gelesen wurden. Vielleicht war Amo dies zu

27 Ludovici, Carl Günter: Entwurf einer vollständigen Historie der wolffischen Philosophie. Teil III. Bd. 1,3. Leipzig 1738, S. 230. Vgl. hierzu kritisch die Dissertation von Edeh, Yawovi Emmanuel: Die Grundlagen der philosophischen Schriften von Amo. In welchem Verhältnis steht Amo zu Christian Wolff, dass man ihn als einen ,führnehmlichen Wolffianer" bezeichnen kann? Essen: Verlag Die Blaue Eule 2003, S. 11 u. passim.

28 Die soeben angeführte Dissertation von Yawovi Emmanuel Edeh bemüht sich vollumfänglich um den wissenschaftlichen Beleg dafür, dass Amo nicht den Wolffianern zuzurechnen sei; vgl. hierzu auch das abschließende Kapitel seines Bandes in ebda., S. 144-162. Die entgegengesetzte Position findet sich u. a. auch bei Abraham, William E.: Anton Wilhelm Amo, S. 195 f. 
Beginn seiner akademischen Laufbahn noch nicht bewusst; später aber dürfte es für ihn ausschlaggebend gewesen sein.

Demgegenüber konnte das Deutsche bestenfalls als eine regionale Sprache gelten, der im Übrigen im weiteren Verlauf des 18. Jahrhunderts innerhalb der sich herausbildenden internationalen Netzwerke der europäischen Aufklärung ihres unbestreitbaren Aufstiegs gerade im Bereich der Philosophie ab der zweiten Jahrhunderthälfte zum Trotz - doch nur eine sekundäre Rolle zufiel. Die Verwendung des Lateinischen hingegen bot zumindest prinzipiell die Chance, von einem spezialisierten Lesepublikum weit über die Grenzen der deutschen Fürstentümer hinaus rezipiert werden $\mathrm{zu}$ können und eine internationale Öffentlichkeit zu erreichen.

So stand die Sprachwahl Amos ohne jeden Zweifel im Zeichen einer überregionalen und auch die Grenzen Europas überspannenden Kommunikationsfähigkeit, die den jungen Afrikaner jenseits seiner Sprachkompetenzen im Bereich des Deutschen, Niederländischen, Französischen, Englischen, Lateinischen, Griechischen oder Hebräischen interessieren musste. Amo war zweifelsohne polyglott, was ihn von den meisten seiner deutschsprachigen Zeitgenossen unterschied. Gerade in der Sonderkonjunktur einer Globalisierung unter holländischen Vorzeichen musste dem Studenten und Doktoranden in Halle, Wittenberg und Jena deutlich vor Augen stehen, dass das Deutsche keine Weltsprache war, das Lateinische - das noch immer erhebliche Kommunikationsaufgaben gerade auch in einem globalen Kontext erfüllte - aber sehr wohl. Amos mündliche wie schriftliche Sprachverwendung stand als Sprechen und Schreiben jenseits der eigenen Muttersprache ohnehin im Zeichen einer translingualen Sprachenquerung, die von Beginn an eine der zahlreichen weitreichenden Folgen seiner Verschleppung aus Westafrika darstellte. Doch auf dieses Schreiben ohne festen Wohnsitz, also translingual jenseits der eigenen Muttersprache, kommen wir später noch ausführlich zurück.

Die Frage der Sprachenwahl musste für einen Menschen, der in einer (Mutter-) Sprache aufgewachsen war, die er niemals beherrscht hatte, zu der er von Deutschland aus keinen Zugang mehr finden konnte und die nur von lokaler und regionaler Bedeutung gewesen sein dürfte, von großer geistiger Tragweite gewesen sein. Dass der Nachname Amo auf die verlorene Muttersprache verwies und damit eine der Zugehörigkeiten signalisierte, zugleich aber auch mit dem Lateinischen in evidenter Beziehung stand, musste für Anton Wilhelm einen wichtigen zusätzlichen Aspekt darstellen. In der definitiven Form des Namens Antonius Guilielmus Amo Afer stehen damit zwei von außen auferlegte Vornamen zwei selbstgewählten Namen und zugleich zwei Sprachen und Kulturen einander gegenüber, welche die ganze Komplexität, aber auch Vektorizität der Situation des jungen Mannes markieren. „What’s in a name?“ Der Name des Philosophen bildete gleichsam sei- 
nen ersten und fundamentalen Text, der sich bewusst in unterschiedliche Traditionen, Sprachen, Kulturen und Herkunftsbezeichnungen einschreibt. Im Namen ist die transareale Dimension von Leben, Denken und Schreiben dieses Philosophen vital (und für alle sichtbar) verankert.

Zugleich ist das Schreiben des ,schwarzen Philosophen“ in einem mehrfachen Sinne ein Schreiben jenseits und in Ermangelung der Muttersprache: ein Gewinn, der auf einem gewaltsam erzwungenen Mangel beruht. Nicht in seiner Muttersprache zu schreiben, stellt freilich im 18. Jahrhundert - gerade auch im Zeichen der sich herausbildenden République des Lettres - keineswegs ein Novum oder gar ein Alleinstellungsmerkmal dar. Anton Wilhelm Amo freilich wusste, dass viele wissen mussten, dass er aus einer im Europa der Frühaufklärung nicht als Kultur wahrgenommenen Sprachgemeinschaft deportiert worden war, um die Sprache und die Sprachen seiner Herren zu erlernen und zu sprechen.

Dass mit diesem Bewusstsein ein besonderes Verhältnis nicht nur zur Sprache, sondern zur Vielsprachigkeit verbunden ist, zeigt sich in seinem Leben wie in seinen Schriften sehr deutlich. Seine nicht allein begriffliche Arbeit an der Sprache war zweifellos auch dem Versuch geschuldet, mehr als „His Master’s Voice“ zu sein und mehr als nur die Sprache des kolonisierten Objekts zu sprechen. Wir können diesen langwierigen Prozess, den Amo durchlief, als ein eigentliches Zur-Sprache-Kommen begreifen, in dessen Verlauf sich Amo als Subjekt seiner eigenen Geschichte zu konstituieren lernte. Einer Geschichte, die aufgrund seines Bildungsweges außerordentlich war und doch zugleich repräsentativ für all jene Schwarze, die als Eigentum und Besitz von Fürsten an deutschen Höfen ihr Leben fristen mussten. Für die Allermeisten unter ihnen gab es ein solches Zur-Sprache-Kommen nicht.

Man muss hier folglich von einem translingualen Spannungsfeld sprechen, in welchem die vektorisierten geographischen Bezugssysteme nach ihrem adäquaten sprachlichen Ausdruck suchten, um ihn schließlich im Lateinischen zu finden, jener ,toten' Sprache aus einer vergangenen Zeit, die seit dem Ausgang des 15. Jahrhunderts wieder zu einer Weltsprache und zur Sprache der Gelehrten geworden war. Amos Arbeit an der Sprache ist freilich niemals monolingual. Selbst dort, wo es um auf den ersten Blick einsprachige Ausdrucksweisen etwa im akademischen Diskurs geht, erscheint doch immer wieder der Bezug zu anderen Sprachen im Text. Dies lässt sich in seiner Wittenberger Dissertation bis hin zur Titelgebung in lateinischer und griechischer Begrifflichkeit beobachten: Dissertatio inauguralis philosophica de humanae mentis APATHEIA seu sensionis ac facultatis sentiendi in mente humana absentia et earum in corpore nostro organico ac vivo praestantia.

Translinguale Bewegungen sind bei Antonius Guilielmus Amo Afer folglich eine Konsequenz transarealer Erfahrungsmuster, die er von Kindheit an auf allen Ebenen seines Daseins durchzuerleben hatte. Die Vielsprachigkeit gab ihm zu- 
gleich aber auch die Möglichkeit zur Entwicklung einer viellogischen Denkweise, einer polylogischen Grundeinstellung, die sich in seinen Schriften immer wieder herausarbeiten lässt. Hieraus entsteht ein überaus komplexes Verhältnis zwischen dem Zur-Sprache-Kommen und dem Zur-Sprache-Bringen, welche das Subjekt Amo charakterisiert. Und dieses Subjekt konstituiert sich translingual, in der Querung verschiedener Idiome, durch Sprache.

Wohl im November 1729, etwa zweieinhalb Jahre nach seiner Immatrikulation, hielt Amo an der Universität Halle unter dem Vorsitz des renommierten Juristen, Diplomaten und Spezialisten für Internationales Recht, dem damaligen Kanzler der Universität, Johann Peter von Ludewig, seine Disputatio zum Thema De iure Maurorum in Europa ab, eine öffentliche Präsentation, mit welcher er sein Studium an der preußischen Universität abschloss. Ludewig, der später auch zu den politischen und diplomatischen Beratern der Könige von Preußen zählte, darf zu den wichtigsten Förderern Amos an der Universität Halle gezählt werden. Er könnte durchaus auf das Thema der öffentlichen Disputatio einen gewissen Einfluss gehabt haben. ${ }^{29}$ Gerade in dieser akademischen Anfangszeit war es für Amo von immenser Bedeutung, über Freunde und Förderer im akademischen Bereich zu verfügen.

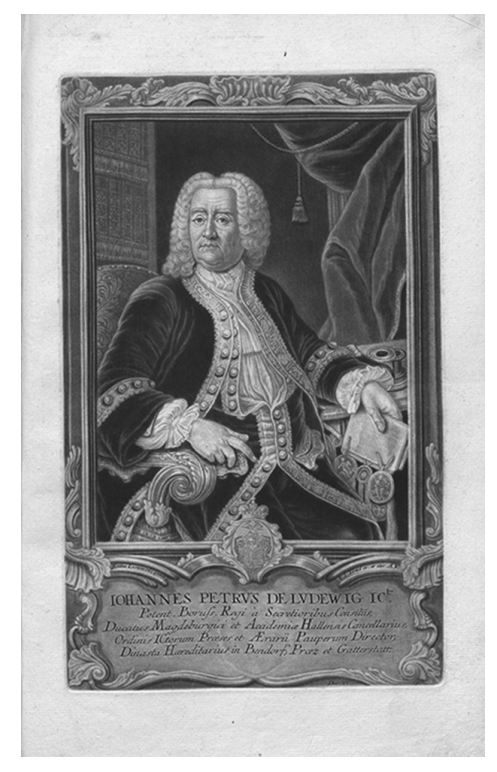

Abb. 19: Johann Jakob Haid: Johann Peter von Ludewig (1688-1743).

29 Vgl. hierzu die sicherlich zu weit gehenden Hypothesen von Suchier, Wolfram: A.W. Amo. S. 444; sowie Lochner, Norbert: Anton Wilhelm Amo. A Ghana Scholar in Eighteenth Century Germany, S. $171 \mathrm{f}$. 
Doch bildete inmitten aller verschiedenen Motivationslagen der gewählte Gegenstand aus dem Bereich der Rechtswissenschaften zweifellos das Thema für den mittlerweile schon erfahrenen und unter seinen Kommilitonen herausragenden Studenten: Es war ihm buchstäblich auf den Leib geschneidert. Denn hier ging es in erster Linie um die rechtliche Stellung jener ,Mohren“ und ,Hofmohren“, jener Afrikaner in Europa, die in einer weitgehend rechtlosen Stellung, gleichsam als „Sklaven ohne Sklaverei“, ${ }^{30}$ in Europa lebten. So ist diese rechtswissenschaftliche Disputation sicherlich nicht nur mit Blick auf die Geschichte des internationalen Rechts in höchstem Maße bemerkenswert. ${ }^{31}$ Es ging um nichts weniger als um eine Rechtsbestimmung, aber auch um eine Selbstbestimmung des in Afrika Geborenen hinsichtlich auch und gerade seiner eigenen Rechte in Europa. Im Kern handelte diese öffentliche Erörterung - und so ließe sich „Disputatio“ auch übersetzen -von nichts anderem als der Klärung der Rechte von Afrikanern in Europa. ${ }^{32}$ Hier verhandelte Amo in eigener Sache! Und er war zur Sprache gekommen, um im juristischen Diskurs zur Sprache bringen zu können, was aufs Engste mit seinem eigenen Lebenswissen ${ }^{33}$ verbunden war - als Mensch von afrikanischer Herkunft im vorfriderizianischen Preußen.

Diese erste Schrift Anton Wilhelm Amos konnte bislang nirgendwo aufgefunden werden und gilt folglich als verschollen. Auch ist noch immer fraglich, ob sie jemals gedruckt worden ist. Darf man hoffen, dass sie jemals von der Amo-Forschung wieder irgendwo ausfindig gemacht werden kann? Wie auch immer: Ihr Thema war brisant! Die Tatsache, dass die Disputation in Halle nachweislich großes Aufsehen erregte, aber selbst in der historiographischen Auflistung aller juristischen Disputationes nicht auftauchte, ${ }^{34}$ mag zusätzlich Erstaunen und nicht von ungefähr einen Verdacht nähren. ${ }^{35}$ Denn hier könnte böse Absicht und eine feindliche Hand im Spiel gewesen sein, um Amos Überlegungen ein für alle Mal wieder aus der Welt zu schaffen. Doch sind auch die Argumente, dass es zu einer Drucklegung dieser

30 Zeuske, Michael: Handbuch Geschichte der Sklaverei. Eine Globalgeschichte von den Anfängen bis zur Gegenwart. Berlin - Boston: Walter de Gruyter 2013, S. 517.

31 Vgl. hierzu Mabe, Jacob Emmanuel: Anton Wilhelm Amo interkulturell gelesen, S. $20 \mathrm{f}$.

32 Vgl. hierzu auch Bess, Reginald: A.W. Amo: First Great Black Man of Letters. In: Journal of Black Studies (Thousand Oaks) XIX, 4 (June 1989), S. 387-393, hier S. 391.

33 Vgl. hierzu Ette, Ottmar: ÜberLebenswissen. Die Aufgabe der Philologie. Berlin: Kulturverlag Kadmos 2004.

34 So weist Wolfram Suchier in seiner frühen, bahnbrechenden Studie darauf hin, dass auch in Christoph Weidlichs Verzeichnis aller juristischen Disputationen zu Halle aus dem Jahre 1789 jeglicher Hinweis auf Amos Disputatio fehlt; vgl. Suchier, Wolfram: A.W. Amo, S. 444.

35 Vgl. hierzu Sephocle, Marilyn: Anton Wilhelm Amo. In: Journal of Black Studies (Thousand Oaks) XXIII, 2 (December 1992), S. 185f; sowie Bess, Reginald: A.W. Amo: First Great Black Man of Letters, S. $390 \mathrm{f}$. 
Schrift niemals gekommen sei, nicht gänzlich von der Hand $\mathrm{zu}$ weisen. ${ }^{36}$ So sind wir hier auf Indizien angewiesen und müssen uns mit dem begnügen, was wir über den Inhalt von Amos frühester Schrift über ihren Titel hinaus wissen.

William E. Abraham betonte, Amo habe mit seinen Überlegungen ein möglichst breites Publikum erreichen und ein Nachdenken über die weitgehend rechtlose Lage von Afrikanern in Europa auslösen wollen. ${ }^{37}$ Sollte dies in der Tat sein Ziel gewesen sein, so dürfte die Verwirklichung dieser Absicht durch das Verschwinden seiner Schrift (sollte diese überhaupt jemals in gedruckter Form vorgelegen haben) zumindest bis in unsere Tage unterbunden worden sein. In jedem Falle gilt, dass Anton Wilhelm Amo zumindest nach aktuellem Kenntnisstand auf die von ihm in De iure Maurorum in Europa entfaltete Problematik in seinen späteren Publikationen nicht mehr zurückgekommen ist. Bedeutet dies, dass er bei seinem Zur-Sprache-Kommen bemerkte, dass er nicht alles zur Sprache bringen durfte?

Über Amos erste Schrift wissen wir dank verschiedener historischer Quellen gar nicht so wenig. In seinen Ausführungen griff Amo auf das Römische Recht ebenso zurück wie auf die Rechtstitel afrikanischer Könige innerhalb des Römischen Reiches. Dies war gewiss notwendig, galt doch - wie im Bereich der Sklaverei - das Römische Recht nach seiner Auffassung noch immer überall dort, wo etwa das Germanische Recht keine näheren Aussagen traf oder Bestimmungen erließ. ${ }^{38}$ Im Kern der Amo'schen Argumentation scheinen deutlich die den Afrikanern zugebilligten Rechtstitel gestanden zu haben, die jeglicher Legitimation von Sklaverei entgegenstanden. Amo berührt damit den juristischen Sachverhalt einer Sklavenschaft ohne Sklaverei.

Der politisch so einflussreiche von Ludewig, der weder als Freund der Wolff'schen Philosophie noch ihrer Vertreter gelten darf, setzte sich für den „Mohren in Europa“ Anton Wilhelm Amo ein. Er förderte ihn auch auf seinem weiteren Weg und unterstützte ihn bis zum eigenen Tod im Jahr 1743. Dies schließt offensichtlich auch die Überlegungen Amos nicht nur zur abstrakten Rechtslage der Afrikaner allgemein, sondern auch zur konkreten Situation des jungen Philosophen selbst mit ein. Alles deutet darauf hin, dass Amo sich auf von Ludewig als Ratgeber und Freund bis in seine Zeit an der Universität Jena verlassen konnte. An die Stelle seiner fürstlichen Herren und Gönner trat damit zunehmend eine Gestalt, die als Universitätskanzler und Rechtswissenschaftler über subtilste Kenntnisse im akademischen Feld verfügte. Ihr Tod sollte später für Amo mehr als schmerzlich sein.

36 Vgl. Brentjes, Burchard: Anton Wilhelm Amo. Der schwarze Philosoph in Halle, S. 37.

37 Vgl. Abraham, William E.: The Life and Times of Anton Wilhelm Amo, S. 430.

38 Vgl. hierzu auch ebda., S. $430 \mathrm{f}$. 
Am Beginn von Anton Wilhelm Amos Schriften steht so eine Selbstbestimmung und mehr noch Selbstreflexion, die es ihm erlaubte, seine eigene Lage und Position, von der er aus sprach und sprechen konnte, klug zu thematisieren, zu diskutieren und mit seiner Person, mit seinem Namen zu verbinden. Das Versuchsobjekt hatte sich nicht nur als intelligent erwiesen, sondern zugleich einen eigenen Versuch in Sachen selbstbestimmten Lebens unternommen. Eine Kippfigur aber ergab sich zwischen der „Freyheit“ und der „Dienstbarkeit“, gerade auch mit Blick auf die „von Christen erkauften Mohren in Europa“. ${ }^{39}$ Damit waren die Grundlagen für die Gewinnung einer vielperspektivischen philosophischen Position gelegt, wie sie in den afrikanisch-europäischen Beziehungen innerhalb der Aufklärungsphilosophie im deutschsprachigen Raum - und nicht nur dort - wohl einzigartig ist. Dass dieser Aspekt später aus der Geschichte der deutschen Philosophie wie der Geschichte der Philosophie in Deutschland wissentlich getilgt werden sollte, unterstreicht nur die Bedeutung dieses verschütteten Denkers der Aufklärung.

Indem er sich in der Folge Fragen zuwandte, wie sie die Philosophie(n) der deutschen Frühaufklärung bewegten, sicherte er sich die Sympathie und auch die Unterstützung vieler Kollegen und mancher Förderer, allen voran des erwähnten Johann Peter von Ludewig. Aus der Reflexion seiner Deportation wie seiner Transplantation in einen von seinem Geburtsland gänzlich verschiedenartigen Kontext entsteht in der sich herausbildenden Philosophie des Antonius Guilielmus Amo eine durch den Zusatz „Afer“ immer wieder neu ins Bewusstsein gerufene spezifische Dimension einer Innerhalb- und Außerhalbbefindlichkeit, welche die Untersuchung insbesondere erkenntnistheoretischer Fragestellungen zweifellos als vordringlich erscheinen ließ. Die afrikanische Herkunft des deutschen Philosophen in Europa reduzierte in der Folge in keiner Weise die anderen Bestandteile seines Namens, in dem sich wie in wohl keinem anderen Text sein Zur-SpracheKommen konzentriert und kristallisiert.

Wie erwähnt, tobte zu dem Zeitpunkt, als Amo von seinen herzoglichen Förderern, aber auch ,Eigentümern' und Herren an die aufstrebende preußische Universität von Halle geschickt wurde, dort noch immer der Kampf der starken pietistischen Fraktion gegen die Rationalisten. Wie ebenfalls bereits kurz angesprochen, hatte ihr wichtigster Kopf, der zwischen Leibniz und Kant sicherlich originellste deutsche Aufklärungsphilosoph Christian Wolff, seine philosophische Position mit der sofortigen Verbannung aus Preußen bezahlen müssen. Das vorfriderizianische Preußen hatte Position bezogen und dies war Amo nicht entgangen. Gleichwohl gab es in Halle eine Vielzahl an Professoren, wie etwa den mit Wolfenbüttel eng verbundenen Christian Thomasius, der sich im Bereich der Jurisprudenz

39 Zit. nach Brentjes, Burchard: Anton Wilhelm Amo. Der schwarze Philosoph in Halle, S. 38. 
für ein humanes, an den Ideen der Frühaufklärung ausgerichtetes Strafrecht einsetzte und im Übrigen wesentliche Akzente in der Leib-Seele-Problematik gesetzt hatte. Diese waren ebenfalls für ihre offenen und freiheitsliebenden aufklärerischen Positionen bekannt. Amo konnte folglich hoffen, dass sich aufklärerische Positionen in Philosophie und Rechtswesen doch noch durchsetzen würden.

Rekapitulieren wir nochmals kurz die damalige Situation der Hallenser Wissenschaftsgemeinde. Auch nach dem Jahre 1723 hielten die oftmals vehementen Auseinandersetzungen und Machtkämpfe unvermindert an, in denen sich insbesondere (der allerdings bereits im September 1728 und damit für Amo sehr früh verstorbene) Christian Thomasius und Johann Peter von Ludewig wiederholten Anfeindungen ausgesetzt sahen. ${ }^{40}$ Anton Wilhelm Amo muss sehr früh begriffen haben, dass sich wissenschaftliche und intellektuelle Positionen niemals in einem abstrakten Raum ansiedeln, sondern die Ergebnisse komplizierter Vermittlungen sind. Er zog daraus Lehren für die eigene Laufbahn.

Mit den ständigen Veränderungen innerhalb dieses keineswegs autonomen, sondern von staatlichen wie klerikalen Eingriffen unmittelbar betroffenen Feldes hing sicherlich auch Amos Entscheidung zusammen, der Universität Halle und damit Preußen vorerst den Rücken zu kehren und an die damals noch nicht mit letzterer verbundene Universität Wittenberg zu wechseln. Angesichts des skizzierten akademischen Spannungsfeldes war dies zweifellos eine gute Entscheidung, der Amo sicherlich auch die glücklichste Zeit an einer deutschen Universität verdankte. ${ }^{41}$ In Halle setzten sich die Auseinandersetzungen zwischen Wolffianern und Pietisten fort, wobei es in der Folge auch zum Weggang mehrerer wichtiger Professoren (wie etwa des bereits erwähnten Ludovici) aus Preußen kam. Die Universität Halle hatte unverkennbar an Bedeutung wie an Ausstrahlungskraft verloren.

Dieser für den Fortbestand der Universität nicht ungefährlichen Abwanderung schloss Amo sich an. Und man könnte im Sinne der Bourdieu'schen Feldtheorie ${ }^{42}$ durchaus davon ausgehen, dass das Verlassen Preußens in gewissem Maße auch dem Verlassen eines zum einen für Preußen, aber auch für die Universität Halle spezifischen Teilfelds philosophischer Positionskämpfe gleichkam. Damit eröffnete sich für Amo eine zweite akademische Spielfläche, auch wenn diese schon aufgrund der Nähe zu Halle nicht grundsätzlich von der dortigen Universität getrennt war. Aber das Überschreiten der preußischen Landes-

40 Vgl. ebd., S. $34 \mathrm{f}$.

41 Vgl. hierzu auch Brentjes, Burchard: Anton Wilhelm Amo. Der schwarze Philosoph in Halle, S. $38 \mathrm{f}$.

42 Vgl. hierzu auch Jurt, Joseph: Das literarische Feld. Das Konzept Pierre Bourdieus in Theorie und Praxis. Darmstadt: Wissenschaftliche Buchgesellschaft 1995. 
grenze bot doch einen zusätzlichen, wenn auch nur momentanen Schutz für die eigene Person vor pietistischen Verfolgungen jeder Art.

So trug sich Amo am 2. September 1730 in die Matrikel der Universität Wittenberg ein, wo die Pietisten zum damaligen Zeitpunkt noch keine vergleichbare Macht zu entfalten in der Lage waren. Bereits wenige Wochen später, am 17. Oktober 1730, wurde er zum Magister der Philosophie und der Freien Künste promoviert: Seine universitären Leistungen aus Halle waren mit einer Schnelligkeit anerkannt worden, die aus heutiger Perspektive Bewunderung, vielleicht auch Scham erregen muss: Sie alle wissen, dass wir in unserem digitalen Zeitalter für Anerkennungen akademischer Leistungen wesentlich länger brauchen. Doch glücklicherweise musste Amo keine übersetzte Geburtsurkunde aus seinem Heimatdorf und kein polizeiliches Führungszeugnis vorlegen. Doch ich erspare mir weitere Kommentare: Selbst eine simple Impfung in Corona-Zeiten verursacht einen wesentlich höheren bürokratischen Aufwand im selben Bundesland als eine damalige Anerkennung akademischer Abschlüsse jenseits der ehemaligen Landesgrenzen! Amos Weggang nach Wittenberg war auch insofern ein geschickter Schritt, als er nun über einen ersten akademischen Abschluss verfügte, auf den er nun an einer weiteren angesehenen Universität aufbauen konnte.

Zugleich veränderte Amo nun sein wissenschaftliches Profil und wandte sich verstärkt den medizinischen Studien zu. Damit legte er die Grundlagen für seine spätere Dissertation, die sich im Grenzgebiet zwischen Medizin, Anthropologie und Philosophie ansiedeln sollte. Unter der fürsorglichen Begleitung des Physikers Martin Gotthelf Loescher, der zu den gemäßigten Mechanisten im Bereich der Medizin zählte, ${ }^{43}$ arbeitete sich der frischgebackene Magister in die Erforschung der neuen Gegenstände zügig ein und erwarb sich große Anerkennung bei den Professoren wie den Studierenden in Wittenberg.

Man darf ohne jede Übertreibung sagen, dass die Universität Wittenberg sich ihres schwarzen Magisters rühmte und ihn anlässlich eines Besuchs des neuen Kurfürsten von Sachsen Friedrich Augusts III., des späteren polnischen Königs, in der Universitätsstadt bewusst an die Spitze der Abordnungen stellte, die den Souverän empfingen. ${ }^{44}$ Binnen weniger Jahre hatte sich Amo ein hohes Prestige in der Universitätsstadt erworben. Es sah gut aus für die akademische Laufbahn des jungen Schwarzen in Deutschland.

43 Brentjes, Burchard. Anton Wilhelm Amo. Der schwarze Philosoph in Halle, S. 41.

44 Vgl. hierzu Lochner, Norbert: Anton Wilhelm Amo: A Ghana Scholar in Eighteenth Century Germany, S. $174 \mathrm{f}$. 
Am 10. Mai des Jahres 1733, so bezeugen es die Hamburgischen Berichte von neuen Gelehrten Sachen vom 2. Juni desselben Jahres, seien die Ehrenformationen in Gegenwart des gnädigen sächsischen Kurfürsten zur Erbhuldigung und Ehrenbezeugung angetreten. Bereits ein kleiner Ausschnitt aus dem detaillierten Bericht mag uns ein anschauliches Bild davon vermitteln, welche Rolle Anton Wilhelm Amo übernahm:

Der Herr M. Amo, ein Africaner, stand in der Mitten, als Commandeur über das gantze Corpo, schwarz gekleidet, einen propren Stock in der Hand tragend, und über die Weste mit einem breiten weissen Ordens-Bande angethan, worauf das Chur-Sächs. Wapen mit Gold und untermengten schwartzen Seide prächtig gestickt war. Die beyden Marschälle aber, welche ihn in das Collegium begleitet, trugen schwartze Ordens-Bänder über das Hemde, damit zwischen ihnen, was den Putz anlanget, ein Unterschied seyn mögte. ${ }^{45}$

Der ,schwarze Philosoph،, der in einem prachtvollen schwarzen Ornat erschien, war endgültig zu einem Vorzeigeobjekt geworden, mit dem man bei offiziellen Gelegenheiten Staat machen konnte. Sicherlich kann man diese Herausstellung des an mehreren Aufzügen zu Ehren des sächsischen Kurfürsten beteiligten und seine Marschälle kommandierenden schwarzen Magisters auch als eine Kippfigur lesen. Dies insofern, als sich die Ausnahmestellung Amos von der Inklusion sehr rasch in ihr Gegenteil einer Exklusion verkehren konnte, mochte sich doch der eine oder andere der erlauchten Zuschauer an die Rolle der Hofmohren gerade auch an deutschen Fürstenhöfen erinnert fühlen. Die herausgehobene Stellung Amos ist folglich durchaus ambivalent zu beurteilen. Und sie sollte sich auch in der Tat mit Blick auf die künftige Rolle des Philosophen in Deutschland als höchst ambivalent erweisen.

Seine Ehrung beruhte zweifellos auf dem hervorragenden Ruf, den sich der junge Magister der Philosophie und freien Künste im Verlauf der zurückliegenden Jahre an der Universität Wittenberg erworben hatte. Und auch sonst scheint der „Africaner“ durchaus in das universitäre Leben integriert gewesen zu sein, bewahrt das Stadtarchiv Wittenberg doch noch Listen auf, in denen - wie der Stadtarchivar Kühne herausfand ${ }^{46}$ - die säumigen Zecher erfasst wurden - unter ihnen kein Geringerer als der Herr Magister Amo. Es gab offenkundig genügend Gründe zum Feiern.

Doch dieser in Wittenberg weithin anerkannte und respektierte angehende Philosoph trug sich auch in eine andere Liste ein: jene der Doktoranden, die ihre

45 Hamburgische Berichte von neuen Gelehrten Sachen (Hamburg) XLIX (2. Juni 1733), S. 366. 46 Hierauf macht aufmerksam Brentjes, Burchard: Anton Wilhelm Amo. Der schwarze Philosoph in Halle, S. 46. 
Dissertationen vorbereiteten und sich im Schuldiskurs deutscher Philosophie in der Zeit der Frühaufklärung übten. Wenn Anton Wilhelm Amo sich in Halle mit einer juristischen Problematik auseinandergesetzt hatte, die für ihn von vitalem Interesse war, so bestand seine List in Wittenberg darin, nunmehr einer erkenntnistheoretischen Fragestellung zu folgen, die sich mit dem Verhältnis von Körper und Seele, mit der Leib-Seele-Problematik, beschäftigte. Bei dieser Thematik, die zweifellos zu den wichtigen, vielleicht sogar zentralen, in jedem Falle stark umkämpften Fragestellungen der damaligen Philosophie gehörte, ging es Amo um eine höchst innovative Sichtweise des Verhältnisses zwischen Körper und Seele, wobei die selbstgestellte Aufgabe darin bestand, die Zurechnung der Empfindungen dem Körper oder der Seele zuzuordnen. Nur auf den ersten Blick erscheint diese Fragestellung als ein rein akademisches Problem. Worin aber besteht das Spannende in ihr?

In seiner vierundzwanzig Seiten umfassenden Inaugural-Dissertation $D e$ humanae mentis apatheia (deren vollständiger Titel zu übersetzen wäre mit Die Apatheia der menschlichen Seele oder über das Fehlen der Empfindung und der Fähigkeit des Empfindens in der menschlichen Seele und das Vorhandensein von beiden in unserem organischen lebenden Körper) ging Amo dieser Frage in lateinischer Sprache auf höchst systematische und konzise Weise nach. Bereits im Titel findet sich dabei das für die Arbeit zentrale Lexem „vivo“. Dieses eminent wichtige Lebens-Lexem wird Amo in seiner straff und klar strukturierten Dissertation nach der Aufzählung zweier negativer Thesen in der dritten, nun affirmativen These in der Titelformulierung gleich auf der ersten Seite wiederholen, wo von „unserem organischen lebenden Körper" die Rede ist. ${ }^{47}$

\footnotetext{
47 Ich benutze hier die deutsche Ausgabe von De humanae mentis apatheia in: Antonius Guilielmus Amo Afer aus Axim in Ghana: Übersetzung seiner Werke. [Übers. A. Blaschka?]. Halle (Saale): Martin-Luther- Universität Halle-Wittenberg 1965, hier S. 71. Diese Übersetzung ist nicht unproblematisch, wie schon die aktualisierende Angabe des Verfassers (aus Ghana, nicht wie im Original aus Guinea) zeigt. Die Originalausgabe in lateinischer Sprache erschien 1734 in Wittenberg bei Schlomacher unter dem Titel Dissertatio inauguralis philosophica de humanae mentis APATHEIA seu sensionis ac facultatis sentiendi in mente humana absentia et earum in corpore nostro organico ac vivo praestantia. Eine etwas differierende Übersetzung findet sich im Anhang zu Brentjes, Burchard: Anton Wilhelm Amo. Der schwarze Philosoph in Halle, S. 87-108. Das Originalzitat in lateinischer Sprache lautet: „corpori nostro organico et uiuo“ (hier wie in der Folge zitiert nach der faksimilierten Edition der Dissertatio inauguralis philosophica de humanae mentis APATHEIA seu sensionis ac facultatis sentiendi in mente humana absentia et earum in corpore nostro organico ac vivo praestantia. Philosophische Inauguraldissertation, Wittenberg 1734, Facsimile-Ausgabe. Halle (Saale: Martin-Luther-Universität Halle-Wittenberg 1978, S. 3.)
} 
Gute Gründe lassen sich dafür ins Feld führen, in dieser Inaugural-Dissertation zumindest in einem weiten Sinne eine lebenswissenschaftlich ausgerichtete Studie zu erkennen. Denn ging es in De iure Maurorum in Europa um eine das eigene Lebenswissen des in Afrika Geborenen und nach Europa Verschleppten integrierende rechtswissenschaftliche Fragestellung, so verfolgte die Dissertation die Frage nach dem Leben aus der Perspektive des Leib-Seele-Dualismus. Die intertextuellen Gesprächspartner waren in dieser Studie nicht nur Verfasser medizinischer oder physiologischer Abhandlungen, sondern auch Philosophen vom Range eines John Locke oder René Descartes, mit denen sich der schwarze deutsche Philosoph kritisch befasste. Denn er hatte die Reichweite seiner philosophischen Studien deutlich ausgeweitet.

Die im Jahr 1734 in Wittenberg vorgelegte, unter dem Vorsitz von Martin Gotthelf Loescher öffentlich verteidigte und noch im selben Jahr im Druck erschienene Inaugural-Dissertation geht dabei davon aus, dass der menschliche Körper „als etwas sehr Vollkommenes aus verschiedenen lebenskräftigen und beseelten Organen zuerst von dem Schöpfer kunstreich verfertigt und auch durch Zeugung fortgepflanzt worden“ ist. ${ }^{48}$ Hier wird der Körper als lebendige, von einem Schöpfer erzeugte, von Menschen gezeugte und von einer Lebenskraft (,vis vitalis“) vorangetriebene Einheit verstanden, wobei sich Amo hier auf eine von ihm angeführte Schrift Christian Vaters stützt.

Die zentrale und für die Aufklärungsperiode wichtige Setzung Amos ist die einer Lebenskraft. Allen Dingen wohnt laut Amo eine solche inne. Der zentrale Begriff der Empfindung wird dabei mit jenem des Lebens verbunden:

Alles, was empfindet, das lebt. Alles, was lebt, nimmt Nahrung auf. Alles, was lebt und Nahrung aufnimmt, wächst. Alles, was von dieser Art ist, wird schließlich in seine Urstoffe (prima principia) aufgelöst. Alles, was in seine Urstoffe aufgelöst wird, ist aus Urstoffen zusammengesetzt (principiatum). Alles, was aus Urstoffen zusammengesetzt ist, hat seine konstitutiven Teile. Alles, was von dieser Art ist, ist ein teilbarer Körper. Wenn also die menschliche Seele empfindet, so folgt daraus, dass sie ein teilbarer Körper ist. ${ }^{49}$

48 Antonius Guilielmus Amo Afer: Übersetzung seiner Werke, S. 76. Im lateinischen Original: „ad corpus quod adtinet est: elegantissimum e diuersibus organisuitablibus et animalibus a creatore primum fabrefactum, et de hinc quoque per generationem propagatum." (De humanae mentis apatheia, S. 9.)

49 Ebda., S. $101 \mathrm{f}$. Im lateinischen Original: „Quidquid sentit, illud uiuit, quidquid uiuit nutritur, quidquid uiuit et nutritur augmentatur, quidquid huius modi est, tandem in sua Prima principia resoluitur, quidquid in sua prima principia resoluitur, est principiatum, omne principiatum habet suas partes constitutiuas, quidquid eius modi est, est corpus diuisibile si igitur mens humana sentit, sequitur quod sit corpus diuisibile.“ (De humanae mentis apatheia, S. 15.) 
Anton Wilhelm Amo verwendet die ganze Schärfe seines Geistes darauf, mit Hilfe seiner Logik und mancherlei Syllogismen zu beweisen, was er bereits im ersten Teil seiner Arbeit, gleich zu Beginn seiner Ausführungen, als Behauptung oder Arbeitshypothese deutlich formuliert hat. Dass nämlich ,jeder Geist außerhalb jedes Leidens steht“"50 - und er bekräftigt, dass wenn etwas materiell sei, „dass es nicht immateriell sein“ könne. ${ }^{51}$ In seinem radikalen Körper-Geist-Dualismus trennt er beide Bereiche folglich scharf voneinander ab.

Folgen wir der Hauptthese der Inaugural-Dissertation, dann ist die menschliche Seele, dann ist der menschliche Geist von allem Materiellen, allem Sinnlichen, allem Körperlichen getrennt und gleichsam ,gereinigt‘ (und damit vom Körperlichen abgezogen, also abstrakt). Man könnte den Körper-Geist-Dualismus Amos, den man wohl einem gemäßigten Mechanismus zuordnen darf ${ }^{52}$ und der eine durchaus deutliche Kritik an den Positionen von René Descartes enthält, ${ }^{53}$ sehr wohl mit einer analogen Abtrennung in Verbindung bringen, die das Seelische, das Geistige vor allem Körperlichen schützt und damit von aller physischen Verfasstheit klar scheidet. Wir werden die Konsequenzen dieser wechselseitigen Abtrennung von Leib und Seele gleich erkennen.

Die Freiheit des Geistes zählt zweifellos zu den zentralen Theoremen der Philosophie Anton Wilhelm Amos. Es ist eine Freiheit, die von jedem Körperlichen abgetrennt ist. Und diese zentrale Überzeugung dürfte sich aus Quellen und einem Lebenswissen speisen, das für Amo höchst spezifisch ist und in seiner lebenspraktisch nicht hintergehbaren Rolle als Mensch schwarzer Hautfarbe unter Weißen begründet liegen dürfte. Insofern transportiert seine Philosophie nicht nur mit Blick auf seine rechtliche Situation, sondern auch auf die zentralen Begriffe zeitgenössischer philosophischer Debatten sehr viel Autobiographisches. Das Denken Amos erklärt sich für frei von jeglicher körperlichen Bindung oder Verfasstheit.

Man könnte aus den Überlegungen Amos auch die Folgerung ableiten, dass die Immaterialität des Geistes zugleich eine Freiheit impliziert, welche eine „apatheia“ im positiven Sinne, also Gleichmut und Seelenruhe, ganz in der Tradition der Stoa ermöglicht. Diese Form der unerschütterlichen Seelenruhe, auf deren

50 Ebda., S. 73. Im lateinischen Original: „Omnem spiritum esse extra omnem passionem.“ (De humanae mentis apatheia, S. 5.)

51 Ebda., S. 74. Im lateinischen Original: „Si aliquid est immateriale sequitur quod materiale esse nequeat.“ (De humanae mentis apatheia, S. 6.)

52 Vgl. hierzu Brentjes, Burchard: Anton Wilhelm Amo. Der schwarze Philosoph in Halle, S. 46. 53 Vgl. hierzu Wiredu, Kwasi: Amo's Critique of Descartes` Philosophy of Mind. In (ders., Hg.): A Companion to African Philosophy, S. 200-206. 
Vertreter Anton Wilhelm Amo in seinem späteren Tractatus de arte sobrie et accurate philosophandi verwies, ${ }^{54}$ kann aber auch eine Waffe im philosophischen Kampf sein, insbesondere dann, wenn das Denken, wenn die Tätigkeit des Geistes, nicht an die materielle, physische Dimension des Körpers zurückgebunden und von dieser abhängig ist. Die Seelenruhe dieser ,Apathie‘ schließt eine Seelengröße in sich ein, die auch den Anspruch darauf erheben kann, unabhängig von der jeweiligen Betrachtung des Körpers ganz selbstverständlich auf gleicher Augenhöhe argumentieren zu können. Auch in Amos Tractatus, auf den später noch zurückzukommen sein wird, taucht die ,apatheia“ bereits im Titel wieder auf, geht es Amo hier doch um ein Philosophieren im Zeichen nicht allein des Akkuraten und Präzisen, sondern auch des „,sobrie“, des Nüchternen, Unerschütterlichen und Seelenruhigen. Es ist beeindruckend, wie viel Stärke und Kraft, aber auch Selbstbeherrschung und Dagegenhalten aus den Überlegungen des jungen, sich profilierenden Philosophen spricht. Es handelt sich um eine Dissertation, welche die Grundlagen des Amo'schen Denkens umschreibt.

Mit De humanae mentis apatheia bewies Anton Wilhelm Amo nicht allein den Erfolg seiner wissenschaftlichen Sozialisation an deutschen Universitäten, sondern auch sein Talent, die Schärfe eigenständigen Denkens mit der Einsicht in die Spielregeln des akademischen Feldes zu verbinden. Amo ist auch im Sinne Bourdieus ein „Homo academicus“ geworden. In der philosophischen Reflexion von Lebenswissen stellte er sein eigenes Überlebenswissen ${ }^{55}$ im Bereich der Philosophie philosophisch ausdrucksstark unter Beweis.

Es sind für Amo wie für Amos Philosophie unzweifelhaft glückliche Jahre. Noch im selben Monat der öffentlichen Verteidigung seiner Dissertation wurde Anton Wilhelm Amo am 16. April 1734 zum „Magister legens“ an der Universität Wittenberg ernannt und zugelassen. Er durfte hoffen, als Wissenschaftler in Deutschland Karriere machen zu können. Denn mit der Verteidigung der Dissertation im Folgejahr standen für Amo - so schien es - alle Türen im akademischen Bereich offen. Martin Gotthelf Loescher, Vorsitzender der Disputation Amos, hielt einmal mehr die herausragenden Fähigkeiten des jungen Mannes fest und verwies gleich zu Beginn seiner Ausführungen auf „Afrika und sein Land Guinea, das ganz weit von uns entfernt liegt und das einst von den Europäern Goldküste genannt wurde

54 Vgl. zur Tradition der Stoa in Amos Denken sowie zu seinem Versuch einer Synthese zwischen Epiktet und dem Christentum u. a. Firla, Monika: Ein Jenaer Stammbucheintrag des schwarzen Philosophen Anton Wilhelm Amo aus dem Jahr 1746. Stuttgart: AfriTüDe Geschichtswerkstatt 2012, S. 9 f sowie 21.

55 Vgl. hierzu Ette, Ottmar: ÜberLebenswissen. Die Aufgabe der Philologie (2004). 
wegen der ungemein reichen Vorkommen von Gold““. ${ }^{56}$ Es ist gewiss kein Zufall, dass auch in den beiden zuletzt genannten Dokumenten die durchlauchten Gönner und Förderer, aber auch Herren von Anton Wilhelm Amo zuvörderst genannt wurden. Ist hier zumeist zwar von Gnade, Güte und väterlicher Milde die Rede, so wirft dies doch ein bezeichnendes Licht auf jene abhängige Lage, in der man den ,schwarzen Philosophen“ sehr wohl wusste. Denn seine Eigentümer und Besitzer brachten sich diskret in Erinnerung. Dennoch hatte Anton Wilhelm Amo allen Grund, die von ihm angestrebte Universitätskarriere zum Greifen nahe zu wähnen und die Hand nach ihr auszustrecken.

Bald schon verdüsterten sich jedoch die Aussichten für den aufstrebenden promovierten Philosophen. Nicht allein, weil im Jahr 1735 der letzte seiner Förderer unter den Herzögen von Braunschweig-Wolfenbüttel, Herzog Ludwig Rudolf, verstarb. Uns nicht allein deshalb, weil im selben Jahr auch Löscher verschied, der Freund und Lehrer Amos. ${ }^{57}$ Für die Veränderung der Situation ausschlaggebend war sicherlich die Tatsache, dass nunmehr auch in Wittenberg der Kampf gegen alle, die als Anhänger der Wolff'schen Philosophie galten, mit zunehmender Vehemenz geführt und in orthodoxen Kreisen allen Ernstes sogar ein „Kanzelverbot für alle Anhänger der Wolff-Leibniz'schen Philosophie erörtert“ wurde. ${ }^{58}$ Fundamentalismen hatten schon immer Konjunktur.

Damit verwandelte sich das akademische Feld für Amo, der in Wittenberg seinem Namen wiederholt den geographischen Zusatz „Afrikaner aus Guinea“ beilegte, erneut und deutlicher als je zuvor in ein gleichsam militärisches Feld von Auseinandersetzungen, die sein materielles Überleben als Philosoph ernsthaft bedrohten. Denn Anton Wilhelm Amo war kein Studiosus mehr: Er lehrte selbst und hatte bereits erste Schüler gefunden. Das Versuchsobjekt war zwar zu einem Vorzeigeobjekt geworden; doch erschien Amo offenkundig manchen nun zunehmend als Bedrohung. Freunde und Förderer, die ihn schützen konnten, wurden rar. Amo war zunehmend auf sich allein gestellt.

Doch er handelte: Er kehrte wieder zurück nach Preußen, um an der Universität Halle in einer zu diesem Zeitpunkt etwas weniger erhitzten Atmosphäre einen zweiten Versuch zu wagen, seine eigenständige, auf seinem Lebenswissen und ÜberLebensWissen aufbauende Philosophie im Rahmen des frühaufklärerischen

56 In deutscher Übersetzung wieder abgedruckt in Antonius Guilielmus Amo: Übersetzung seiner Werke, S. 87. Im lateinischen Original: „Africam \& ejusdem longissime a nobis dissitam regionem Guineam, olim ora aurea, ob copiosissimum auri proventum ab Europaeis appellatam“ (De humanae mentis apatheia, S. 23.)

57 Vgl. Brentjes, Burchard: Anton Wilhelm Amo. Der schwarze Philosoph in Halle, S. 47. 58 Ebda. 
Diskursuniversums weiterzuentwickeln. Preußen schien ihm hierfür immer noch verlockend. Er wusste, dass er sich weiterqualifizieren und als Philosoph eine umfangreichere Schrift vorlegen musste, wollte er seine noch immer bestehende Chance wahrnehmen, seinen Traum von einer akademischen Laufbahn an deutschen Universitäten fortzusetzen. Es gab zu diesem Zeitpunkt keinen Grund, diese Zielstellung, die er nun schon über Jahre verfolgt hatte, unvermittelt aufzugeben. So setzte er seine Hoffnungen erneut auf Preußen.

Amo hatte seine universitäre Laufbahn im preußischen Machtbereich zu einem Zeitpunkt begonnen, ${ }^{59}$ zu dem die seit Anfang der 1680er Jahre entstandene „Brandenburgisch-Afrikanisch-Amerikanische Kompanie“ auch weiterhin die Verschiffung deportierter afrikanischer Sklaven von der sogenannten Goldküste in den karibischen Raum unter brandenburgischer beziehungsweise preußischer Flagge betrieb. Dieses Faktum bildet einen höchst widersprüchlichen Hintergrund für eine Karriere, die aus den unterschiedlichsten Perspektiven als emanzipatorisch anzusehen war. Anton Wilhelm Amo stand mit seinem Lebenslauf für diese Emanzipation.

Erst 1738, nach der Deportation von mindestens 30000 Afrikanern, liquidierte der „Soldatenkönig“ die aufgrund schlechter Geschäfte längst in Schwierigkeiten befindliche Handelsgesellschaft, deren Auflösung somit noch vor den Tod von König Friedrich Wilhelm I. am 31. Mai 1740 und vor die Thronbesteigung seines Sohnes Friedrich II. von Preußen fällt. In diesem Preußen also, dessen kolonialistische Träume nach dem ersten Drittel des 18. Jahrhunderts vorerst ausgeträumt schienen, nimmt sich die in wachsendem Maße registrierte Präsenz des aufstrebenden und erfolgreichen, nun deutlich über dreißig Jahre alten Philosophen wie die inverse Darstellung einer Gesellschaft aus, die in ihren kolonialistischen Ambitionen gescheitert war. Vor dem Hintergrund kolonialer Träume Preußens und der Verwicklungen des Landes in den transatlantischen Sklavenhandel erscheint die Figur des Anton Wilhelm Amo geradezu als Provokation.

Der nach Leibniz im deutschsprachigen Raum zweifellos größte Philosoph, Immanuel Kant, in dessen Werk sich anders als bei seinem Zeitgenossen Blumen-

59 Vgl. Lennert, Gernot: Kolonisationsversuche Brandenburgs, Preußens und des Deutschen Reiches in der Karibik. In: Carreras, Sandra / Maihold, Günther (Hg.): Preußen und Lateinamerika. Im Spannungsfeld von Kommerz, Macht und Kultur. Münster LIT-Verlag 2004, S. 11-23; sowie Weindl, Andrea: The Slave Trade of Norther Germany from the Seventeenth to the Nineteenth Centuries. In: Eltis, David / Richardson, David (Hg.): Extending the Frontiers: Essays on the New Transatlantic Slave Trade Database. New Haven: Yale University Press 2008, S. 250-271. Zur Quellenlage vgl. Jones, Adam: German Sources for West African History. Wiesbaden: Franz Steiner Verlag 1983; sowie (ders.): Brandenburg Sources for West African History 1680-1700. Stuttgart: Franz Steiner Verlag 1985. 
bach keinerlei Verweis auf Amo zu finden scheint, sollte Jahrzehnte später zu einer wichtigen Legitimationsfigur nicht nur des Rassedenkens, sondern rassistischer Vorstellungen werden. Diese schrieb der Königsberger Philosoph gerade mit Blick auf die schwarze Bevölkerung in Afrika und anderswo in seinen Schriften nieder. ${ }^{60}$ Sollte er in seinem Preußen wirklich niemals etwas von dem in Halle, Wittenberg und Jena lehrenden deutschen Philosophen aus Afrika, der auch auf internationale Resonanz gestoßen war, vernommen haben? Dies ist alles andere als wahrscheinlich: Immanuel Kant war der sicherlich prominenteste all jener, die Anton Wilhelm Amo ebenso zu dessen Lebzeiten wie auch lange nach seinem Tode buchstäblich totschwiegen.

Umgekehrt dürfte die Geschichte des brandenburgisch-preußischen Kolonialismus Anton Wilhelm Amo sicherlich sehr vertraut gewesen sein, auch wenn sich in seinen Schriften dazu keinerlei Aussagen finden lassen. Wie hätte er von diesen Aktivitäten nicht wissen sollen, die sich überdies in einem geographischen Raum ansiedelten, $\mathrm{zu}$ dem auch die Region des zwischen verschiedenen europäischen Kolonialmächten aufgeteilten afrikanischen Küstensaums gehörte, in welchem er selbst zur Welt gekommen war? Amo war sich zweifellos der antikolonialen Tragweite und Sprengkraft seiner schieren Existenz und mehr noch seiner akademischen Brillanz bewusst.

Im Dekanatsbuch der Philosophischen Fakultät der Universität Halle ist jener Vermerk zu finden, mit dem am 21. Juli 1736 die Zulassung von Anton Wilhelm Amo nunmehr als Dozent an seiner Alma Mater bestätigt wurde. ${ }^{61}$ Eine für den Philosophen wichtige Rolle innerhalb der wieder veränderten, für die Wolffianer offeneren Situation dürfte dabei erneut der Kanzler der Universität, Johann Peter von Ludewig, gespielt haben, zu dem Amo sicherlich die Verbindung aus dem nahen Wittenberg gehalten hatte. So konnte Amo unter dem Schutz von Ludewigs in Halle seine Tätigkeit als Dozent fortführen, die er - wie wir sahen - mit offenbar so großem Erfolg bereits in Wittenberg ausgeübt hatte. Der Akademiker

60 Vgl. hierzu u. a. Arndt, Susan: Die 100 wichtigsten Fragen: Rassismus, S. 61-63; sowie vor dem Hintergrund der Beschäftigung mit Amo u. a. Gordon, Lewis R.: An Introduction to Africana Philosophy. Cambridge: Cambridge University Press 2008, S. 37f; Mougnol, Simon: Amo Afer. Un Noir, professeur d'université, en Allemagne au XVIIIe siècle. Paris: L'Harmattan 2007, S. 25-27; sowie auch die angesichts der diesbezüglich einschlägigen Kant-Zitate aufgebrachten Äußerungen in der Amo nur kurz behandelnden Publikation von Schilling, Georg: „Who was Anton Wilhelm Amo?" - „Remapping the Jungle“ vs. „Vom faulen Holze lebend“?! An attempt against forgetting and „white“-washing, sad, racist „(a)e(sthe)tic(al)“ texts of so called German „prime fathers“ et al. München: GRIN Verlag 2009, S. 24 und passim.

61 Dieser Vermerk findet sich abgedruckt in Brentjes, Burchard: Anton Wilhelm Amo. Der schwarze Philosoph in Halle, Faksimile zw. S. 47/48. 
wusste aufgrund seiner bedrückenden finanziellen Verhältnisse, die er in seinem Antrag an die Philosophische Fakultät sehr wohl erwähnte, ${ }^{62}$ nur allzu gut, in welch umkämpftem Feld er sich bewegte. Doch war er gewillt, für sein Leben und Überleben im akademischen Milieu weiterzukämpfen.

In dieser Etappe seines Lebens freilich musste der Philosophus in erster Linie darauf bedacht sein, sein im engeren Sinne philosophisches Oeuvre entscheidend zu erweitern. Beharrlichkeit und Fleiß zählten neben der Klugheit zweifellos zu den Charakterzügen und Eigenschaften des ambitionierten Hochschullehrers. Und so konnte im Jahr 1738 - also just in jenem Jahr, als die preußische Krone aus dem transatlantischen Sklavenhandel ausstieg - in Halle sein eigentliches philosophisches Hauptwerk erscheinen: Antonii Guilielmi Amo Guinea-Afri, philosophiae et artium liberalium magistri, Tractatus de arte sobrie et accurate philosophandi. Weder im Vorfeld noch im Nachgang seiner Publikation scheint dieses Werk jedoch von jenen Auseinandersetzungen verschont worden zu sein, die Amo bereits zum Wechsel nach Wittenberg gedrängt, ja gezwungen hatten und ihn im Folgejahr 1739 erneut zum Verlassen der Universitätsstadt Halle bewogen haben dürften. Das ehemalige Vorzeigeobjekt wurde immer stärker in die Defensive gedrängt und sah zunehmend seine Chancen schwinden, eine glänzende akademische Laufbahn in Preußen, Sachsen oder anderen deutschen Ländern mit einer festen Professur krönen zu können.

Dass der Tractatus de arte sobrie et accurate philosophandi ${ }^{63}$ im Lager der Pietisten kaum positiv aufgenommen werden konnte, zeigt sich bereits im paratextuellen Bereich dieses Werkes. Dort bedankt sich der Verfasser in seiner umfangreichen und auf den 6. Juli 1737 datierten Widmung nicht allein bei Johann Peter von Ludewig, sondern auch bei anderen Vertretern eines rationalistischen, kirchlichen Zwängen nicht unterworfenen und deutlich antipietistischen ${ }^{64}$ Philosophieverständnisses höchst wortreich, wobei er seine Dankbarkeitsbeweise und Ehrerbietungen mit einem denkwürdigen Bescheidenheitstopos abschloss:

62 Vgl. Brentjes, Burchard: Anton Wilhelm Amo. Der schwarze Philosoph in Halle, S. 50.

63 Amo, Anton Wilhelm: Tractatus de arte sobrie et accurate philosophandi. Halle: Kitler 1738; ich greife zurück auf die Übersetzung ins Deutsche unter dem Titel Traktat von der Kunst nüchtern und sorgfältig zu philosophieren. In: Antonius Guilielmus Amo Afer: Übersetzung seiner Werke, S. 103-281. Die lateinischen Originalzitate sind in der Folge jeweils entnommen dem Tractatus de arte sobrie et accurate philosophandi. Antonii Guilielmi Amo Guinea-Afri, Philosophiae et artium liberalium magistri. Nendeln: Kraus Reprint 1971.

64 Vgl. hierzu auch Glötzner, Johannes: Anton Wilhelm Amo. Ein Philosoph aus Afrika im Deutschland des 18. Jahrhunderts. Vortrag anlässlich der 500-Jahrfeier der Universität Wittenberg-Halle am 27. Juni 2002 in Halle. München: Editionen Huber 2002, S. 14. 
Wollen Sie daher diese meine wie immer gearteten Versuche, mich um die Öffentlichkeit verdient zu machen, freundlich aufnehmen und Nachsicht üben, sollte mir dabei etwas Menschliches zugestoßen sein; das ist's, worum ich inständig bitte. Im Übrigen möge Gott Sie, meine Mäzene, für beide Gemeinwesen, den Staat und die Gelehrtenrepublik, um die beide Sie sich auf das beste verdient gemacht haben und bis zur Stunde verdient machen, bis in die spätesten Tage in vollkommenem Wohlstand erhalten und noch viele Jahre zurücklegen lassen. Leben Sie wohl und bleiben Sie mir gewogen! ${ }^{65}$

Ein Dank mit Abschied? Dies mag erstaunen. Doch erstmals wird eine veröffentlichte Schrift von Anton Wilhelm Amo nicht mehr mit dem Verweis auf seine Gönner, Herren und ,Eigentümer‘, die Herzöge von Braunschweig-Wolfenbüttel, ,gerahmt', sondern mit der Nennung jener Förderer eröffnet, die Amo - und dafür dürfte er gute Gründe gehabt haben - hier als seine Mäzene bezeichnet. Damit ist zweifellos eine deutlich markierte Eigenständigkeit verbunden, welche die (versklavte) Dienstbarkeit hinter sich lässt und sich bewusst zum Bereich eigener Freiheit bekennt.

Hierbei macht Amo zwei unterschiedliche Felder auf, die er mit den Bezeichnungen „Staat“ und „Gelehrtenrepublik“ belegt, wobei die Autonomie der letzteren - wie Amo sehr wohl wusste - nur eine höchst relative und stets prekäre sein konnte. Er wusste nur zu gut, was selbst einer so renommierten Figur wie Wolff widerfahren war. Doch wird hier unverkennbar signalisiert, dass sich der Verfasser explizit als Afrikaner aus Guinea in dieser Gelehrtenrepublik, dieser République des Lettres, frei bewegt und nunmehr frei seine eigenen Wege wählen will: Gerade auch als Afrikaner aus Guinea! Hieran lässt er keinen Zweifel, im Bewusstsein der Tatsache, wie prekär seine Situation nicht nur im finanziellen Bereich geworden war. Zeichnet sich hier nicht deutlich die Intention Anton Wilhelm Amo Afers ab, dass Philosophie nicht länger das ,Eigentum“ der Europäer sein kann? Wird nicht deutlich der Anspruch erhoben, jenseits aller rassedenkerischen Vorurteile und Grenzziehungen philosophieren zu dürfen, unabhängig von körperlichen Attributen?

Hatte sich Amo in De iure Maurorum in Europa einer juristischen und in De humanae mentis apatheia einer anthropologisch-medizinisch-philosophischen Fragestellung bedient, so bewegt er sich in seinem Traktat über das Philosophie-

\footnotetext{
65 Amo, Anton Wilhelm: Traktat, S. 106. Im lateinischen Original lautet diese Passage: „Quare vt qualescunque hos meos, de publico bene merendi conatus, serena fronte accipiatis, \& quidquid humani passus fuerim ignoscatis, est, quod vehementer rogo. Caeterum Deus Vos, Maecenates, rei vtrique, publicae ciuili, ac literariae, de que vtraque optime meriti estis, \& adhuc meremini, diutissime sospites seruet, pluresque annos perennare faxit. Valete \& fauete!“ (Tractatus, o.P.)
} 
ren, in seinem Tractatus de arte sobrie et accurate philosophandi, von Beginn an auf dem genuin philosophischen Gebiet der Logik. Dabei geht er in einem seiner Arbeit vorangestellten allgemeinen Teil von Frage und Begrifflichkeit der Intention aus und verbindet sie mit ihrer Beziehung zu Gott, aber auch und gerade der menschlichen Seele, ${ }^{66}$ so dass er in seinem Tractatus im Grunde unmittelbar an seine Inaugural-Dissertation wieder anzuknüpfen vermag. Der Bewegungskoeffizient der Amo'schen Philosophie ist in thematischer, gegenständlicher und methodologischer Hinsicht hoch; doch lassen sich sehr wohl die starken Kontinuitäten seiner Kunst des Philosophierens erkennen. Der Tractatus bildet sicherlich deren bisherigen Höhepunkt.

Auf welche Weise Amo in seinem Traktat die mobilen, beweglichen Elemente seiner Philosophie verstärkte, bemerkt man, wenn man sich dem Abschnitt über „Die intellektuellen Ideen“ nähert. Dort heißt es, die Idee sei „entweder gewiß oder wahrscheinlich oder möglich“ ${ }^{67}$ Dabei wird die wahrscheinliche Idee einer Erkenntnis zugeschlagen, „die auf einer Beweisführung von Ähnlichem auf Ähnliches beruht, die immer mit Zweifel verbunden“ sei. ${ }^{68}$ Die mögliche Idee aber „ist

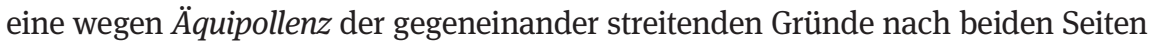
disputable Erkenntnis“. ${ }^{69}$ Was ist unter einer solchen Äquipollenz zu verstehen?

Die Äquipollenz bezeichnet eine Gleichmächtigkeit, meint das GleichvielGeltende, zielt damit - wenn man so will - auf eine Gleich-Gültigkeit, die aber nicht mit Gleichgültigkeit zu verwechseln ist. Dieser aus der traditionellen Logik stammende Begriff, den Amo selbstverständlich nicht erfunden und der ebenso in den Rechtswissenschaften wie den Sprachwissenschaften Verwendung gefunden hat, ist für das Philosophieren im Tractatus de arte sobrie et accurate philosophandi von großer Bedeutung. Dies insofern, als hier äquipollente Begriffe als das Disputable schlechthin jeweils unterschiedliche Blickwinkel und Sichtweisen auf einen Gegenstand zulassen und damit ein Moment des Oszillierens in ein Denken integrieren, das sich seines Standorts, seines Gesichtspunktes nicht (mehr) sicher sein kann. Oder anders: eines Denkens, das sich der gleichen Gültigkeit unterschiedlicher Perspektiven, unterschiedlicher Traditionen, unterschiedlicher

66 Ebda., S. 107.

67 Ebda.; im lateinischen Original: „Idea alia certa, alia probabilis, alia possibilis.“ (Tractatus, S. 79.)

68 Amo, Anton Wilhelm: Traktat, S. 170; im lateinischen Original: „Cognitio consistens in argumentatione a similibus ad similia, Semper cum dubitatione.“ (Tractatus, S. 79.)

69 Ebda., S. 171; im lateinischen Original: „Cognitio in utramque partem disputabilis, ob aequipollentiam rationum pugnantium inter se.“ (Tractatus, S. 79.) 
Blicke bewusst ist. Dies aber ist angesichts des Lebensweges Amos ein fürwahr fundamentaler Gedanke.

Wenn damit neben das Gewisse und das Wahrscheinliche im Bereich der intellektuellen Ideen bei Amo auch das Mögliche tritt, so tritt dieses nicht einfach als ein Hinzugefügtes auf, sondern als das Disputable schlechthin, in welchem ein Gegenstand aus unterschiedlichen Blickrichtungen zugleich und durchaus gleichwertig konstruiert und analysiert werden kann. Ist aus dieser Sicht aber dann nicht auch der Begriff „Theologie“ ein äquipollenter in dem Sinne, dass man ihm aus einer religiösen und kulturellen Verschiedenheit heraus auch unterschiedlichen Sinn und Bedeutung - um hier die Frege'sche Begrifflichkeit einzuführen $^{70}$ - zubilligen müsste?

Amo bedient sich hier spezifischer Begriffe der Logik nicht nur, um terminologische Definitionen zu fixieren, sondern auch, um die Möglichkeit der Engführung verschiedener Blickpunkte und letztlich auch verschiedener religiöser sowie kultureller Logiken einzuführen. Dies ist der erste Schritt zu einem multiperspektivischen Denken. Dabei werden die äquipollenten Terme als Zwillingsbegriffe auf den ersten Blick durchaus stark miteinander verstrebt, zugleich aber auch mit dem Begriff der Fiktion in Verbindung gebracht: „Die Zwillingsform der möglichen Idee ist die fiktive; sie ist eine andere Vorstellung eines Dinges, als es an sich ist. [...] Die Fiktion selbst erfolgt in doppelter primärer Weise: indem geistig zusammengesetzt und getrennt wird, hinsichtlich des Ausspruchs bejaht und verneint wird. “71

Ohne an dieser Stelle die von Amo ausgeführte ,Ideenlehre، weiter verfolgen zu können, dürfte doch deutlich geworden sein, dass gegenüber der Inaugural-Dissertation De humanae mentis apatheia Elemente eines beweglicheren, gleichsam freieren Philosophierens in den Tractatus de arte sobrie et accurate philosophandi eingeführt wurden. Es handelt sich um Elemente und Philosopheme, die - wie es das Beispiel von Amos Definition der Theologie gleich zeigen wird - nicht ohne klare Gefährdungspotentiale für den Philosophen selbst bleiben konnten. Denn dieser Traktat behandelt nicht allein Leitlinien der Vorlesungen und Veranstaltun-

70 Vgl. hierzu die sprachphilosophischen Überlegungen von Frege, Gottlob: Über Sinn und Bedeutung. In: Zeitschrift für Philosophie und philosophische Kritik (Halle / Saale) NF 100 (1892), S. 25-50; wieder abgedruckt in (ders.): Funktion, Begriff, Bedeutung. Fünf logische Studien. Herausgegeben und eingeleitet von Günther Patzig. Göttingen: Vandenhoeck \& Ruprecht 1962, S. 38-63.

71 Amo, Anton Wilhelm: Traktat, S. 171; im lateinischen Original: „Gemina possibili idea ficta, quae: Repraesentatio rei aliter ac secundum se est. [...] Fictio ipsa duplici sit modo primario, componendo \& separando mentaliter, affirmando \& negando qua enunciationem.“ (Tractatus, S. 79.) 
gen Amos, sondern stellt eine umfangreiche philosophische Schrift dar, die mit einer gewissen zeitlichen Verzögerung im Jahr 1738 in Halle erscheinen konnte.

Anton Wilhelm Amo legte damit zweifellos eine mutige, selbstbewusste (da sicherlich auch möglicher Gefahren sich bewusste) Veröffentlichung vor, in der die Äquipollenz so etwas wie das Wasserzeichen einer sich unterschiedlichen Logiken gegenüber öffnenden Prozessualität der Philosophie darstellt. Der Tractatus de arte sobrie et accurate philosophandi steht für ein sich abzeichnendes Philosophieren ohne festen Wohnsitz, mit dem der sich selbst insistierend (und geradezu obsessiv) als „Afrikaner aus Guinea“ bezeichnende deutsche Philosoph gewiss auch zunehmend das Risiko in Kauf nahm, in einem ganz konkreten Sinne und mit Blick auf seine Alma Mater in Preußen buchstäblich wohnsitzlos zu werden. Diese Zeit kam schneller, als sich Amo dies wohl hätte vorstellen können.

Im Kontext von dessen Schriften weist auch dieses Werk eine ähnlich klare und konzise Struktur auf, wie dies schon in De humanae mentis apatheia zu beobachten gewesen war. Auch hier zeigt sich die klar erkenntnistheoretisch ausgerichtete Blickrichtung Amos, die in der Regel von der terminologischen Klärung und danach der begrifflichen Entfaltung ihres jeweiligen Terms ausgeht. Wie in De iure Maurorum in Europa und in De humanae mentis apatheia lässt sich auch im Tractatus ein optimistisches Menschenbild erkennen, das überdies von der Wirkkraft des Denkens, von der Deutungsmacht der Philosophie fraglos überzeugt ist. Wie bereits erwähnt, war Amo von Wolffianern zeitgenössisch als Wolffianer bezeichnet worden. Und auch Burchard Brentjes (im Verein mit vielen, die ihm darin nachfolgten) pflichtete dieser Einschätzung in seiner Monographie bedingungslos bei. ${ }^{72}$ Doch sollte man unbedingt zur Kenntnis nehmen, dass gerade mit Blick auf die expliziten intertextuellen Relationen Amo sehr wohl - wie Yawovi Emmanuel Edeh ${ }^{73}$ in der ersten dem Philosophieren Amos gewidmeten Dissertation zeigte - eine deutliche Distanz gegenüber dem ihm zweifellos vertrauten Denken Christian Wolffs (und sei es aus feldtaktischen Gründen) einnahm. Anton Wilhelm Amo präsentiert sich in seinem Traktat als eigenständiger Denker, der zwar Mäzene, aber keine ,Vor-Denker‘, keine eigentlichen Diskursbegründer kennt. Spätestens mit dem Tractatus beschreitet er philosophisch seinen eigenen Weg.

Freilich sind dabei stets die Schranken der kirchlichen Lehre auszumachen nicht im Sinne einer pietistischen Orthodoxie, aber doch eines Anspruchs des Christentums auf diskursive Setzung und Rahmung aller Philosophie. Die Über-

72 Auch mit Blick auf den Tractatus, vgl. Brentjes, Burchard: Anton Wilhelm Amo. Der schwarze Philosoph in Halle, S. $52 \mathrm{f}$.

73 Vgl. Edeh, Yawovi Emmanuel: Die Grundlagen der philosophischen Schriften von Amo, u. a. S. $163 \mathrm{f}$. 
schneidungsbereiche der Felder Philosophie und Theologie waren häufig zu Ausgangspunkten hitziger Auseinandersetzungen nicht zuletzt in Halle geworden, so dass es als höchst mutig, vielleicht sogar als verwegen oder halsbrecherisch gelten darf, wenn sich Amo gerade auf diesem Feld in Abschnitt IX des ersten Kapitels seines Hauptwerkes mit einer Definition der Theologie aus der Deckung wagte. Dort heißt es nach einer ersten, heilsgeschichtlich fundierten Definition der ,christliche[n] Theologie“ als „Habitus der intellektuellen und effektiven Intention, kraft dessen wir uns mit der echten und soliden Erkenntnis der Wahrheit befassen“"74 unvermittelt: „Ich sage ausdrücklich: Die Theologie der Christen. Es gibt nämlich außerdem eine Theologie der Heiden, der Türken usw., ferner je nach Verschiedenheit der Völker.“75

Das klang wie Wolffs Öffnung gegenüber dem Konfuzianismus. Damit stellte Anton Wilhelm Amo nicht nur den alleinigen Anspruch des Christentums auf eine (von Gott selbst begründete) Theologie in Frage, sondern eröffnete auch, wenn auch nur blitzartig, einen Ein- wie Ausblick auf eine Verschiedenartigkeit der Religionen sowie eine Verschiedenartigkeit der Kulturen, ja erkannte die „Verschiedenheit der Völker“76 in einer Formulierung ohne jede Abwertung dieser Völker an. Die Philosophie Anton Wilhelm Amos lässt sich daher in eine Entwicklungslinie einreihen, die man - keineswegs nur im Bereich der Literaturen der Welt - als ein ZwischenWeltenSchreiben ${ }^{77}$ bezeichnen könnte. Wir können sehr wohl mit Blick auf diesen Philosophen und im Rahmen der historisch gegebenen Möglichkeiten von einer Philosophie ohne festen Wohnsitz sprechen, die sich in seinen Arbeiten auszuprägen begann. Dies ist mit Blick auf die Entwicklung der europäischen Aufklärung im 18. Jahrhundert etwas ganz Besonderes.

Denn eine solche Philosophie ist nicht einem einzigen Ort, einer einzigen Perspektive verpflichtet und zuzuordnen, sondern entfaltet sich auf Ebene der bislang genannten Spannungsfelder auf eine zutiefst vektorielle Weise. Sie lässt die Blickpunkte anderer auf dieselben Gegenstände hervortreten und macht seit De iure Maurorum in Europa immer wieder deutlich, dass sie Europa aus seinem Span-

74 Amo, Anton Wilhelm: Traktat, S. 117; im lateinischen Original: „Habitus intellectivae \& effectivae intentionis, quo versamur circa veram \& solidam veritatis cognitionem.“ (Tractatus, S. 15.) 75 Amo, Anton Wilhelm: Traktat, S. 118; im lateinischen Original: „Notantur dico, Theologia Christianorum. Alia enim Theologia Gentilium, alia Turcarum, alia \& alia pro diversitate Gentium.“ (Tractatus, S. 15.)

76 Amo, Anton Wilhelm: Traktat, S. 118; im lateinischen Original: „pro diversitate Gentium.“ (Tractatus, S. 15.)

77 Vgl. zur Entfaltung dieses Begriffs Ette, Ottmar: ZwischenWeltenSchreiben. Literaturen ohne festen Wohnsitz (ÜberLebenswissen II) (2005). 
nungsverhältnis zu anderen Kontinenten, zu anderen Religionen, zu anderen Völkern und Kulturen begreift. Das Projekt der Amo'schen Philosophie, so rudimentär es auch immer in den auf uns gekommenen Schriften ausgebildet sein mag, erlaubt es durchaus, die Differenzen innerhalb einer keineswegs notwendig auf Europa begrenzten République des Lettres zu denken und sie auf einen Horizont zu beziehen, der sich letztlich auf die Existenz der Philosophien der Welt hin entgrenzt. Amos Philosophie(ren) hatte an diesem Punkt des Tractatus zweifellos einen spannenden und zukunftsweisenden Punkt erreicht, der sich für seinen Verfasser aber auch als höchst spannungsgeladen und gefährlich erweisen konnte. Denn für die im Kern polylogische Anlage seines Philosophierens, für ein Denken der Differenz, das im Entstehen war, gab es im Europa der Aufklärung eng gesetzte Grenzen.

Dass Anton Wilhelm Amo das preußische Halle trotz aller ihm durchaus entgegengebrachten Wertschätzung dennoch ein zweites Mal verließ, hat seine Ursachen gewiss nicht in einer mangelnden Qualifikation in den Bereichen Forschung oder Lehre. Vielmehr ist dieser Aufbruch wohl vor allem mit dem sich wieder verschlechternden Umfeld zu begründen, in dem Amos prekäre Situation für diesen unmittelbar gefährlich werden konnte. Das Ziel bei seinem zweiten Auszug aus Halle war diesmal aber nicht mehr Wittenberg, sondern die Universität von Jena: und damit jenseits der bisherigen Ortswechsel eine Bewegung, die keinen festen Bezugspunkt, keinen festen Wohnsitz mehr kennt. Nach Halle sollte der Philosoph nicht mehr zurückkehren, nach Preußen wohl schon. Die letzte Phase des uns bislang bekannten Lebensweges von Anton Wilhelm Amo in Deutschland hatte begonnen.

Am 27. Juni 1739 stellte er seinen im Original erhaltenen ${ }^{78}$ Antrag auf Nostrifikation an der Universität Jena. Es handelt sich um ein mit „Anton Wilhelm Amo, ein Afrikaner, Magister und Dozent der Philosophie und der freien Künste und Rechte Kandidat“ unterzeichnetes Gesuch, das mit der Bitte um Stellungnahme in der dortigen Fakultät rasch in Umlauf gesetzt wurde und bereits am 8. Juli 1739 zu einem positiven Bescheid führte. Ich verkneife mir an dieser Stelle jede Bewunderung für eine solche Schnelligkeit, die an heutigen Fakultäten undenkbar wäre. So konnte Amo bereits wenige Tage später, am 17. Juli desselben Jahres, seinen selbstverständlich in lateinischer Sprache abgefassten Vorlesungsanschlag universitätsöffentlich aushängen. Das Programm der angekündigten Veranstaltung ist überaus breit gefächert und bildet im Grunde zentrale Teile der wissenschaftlichen Aktivitäten des Philosophen ab. Dass sich Amo hierbei aber auch auf das

78 Abdruck des Faksimiles in Brentjes, Burchard: Anton Wilhelm Amo. Der schwarze Philosoph in Halle, S. 60. 
Gebiet der Kryptographie wagte, kann angesichts seiner Erfahrung im kryptographischen Schreiben nicht wirklich überraschen.

Seine kommentierte Vorlesungsankündigung veröffentlichte Amo damit an jener Universität aus, die in Halle noch immer als „der Ort „wolffianischer Verderbnis“ der königlich-preußischen Landeskinder“ galt. ${ }^{79}$ Man kann vermuten, dass sich Amo in Jena ein für seine Anliegen wie für seine Person offeneres geistiges Klima erhoffte. Sein Hinweis auf seine eigene finanziell prekäre Lage und das Fehlen jeglichen Verweises auf die Herzöge von Braunschweig-Wolfenbüttel machen noch einmal deutlich, dass seine Situation nicht mehr mit jener des jungen Studenten und angehenden Magisters vergleichbar war. Denn der Status einer (zumindest Sklaverei-ähnlichen) Dienstbarkeit bestand in sichtbarer Weise nun nicht mehr. Die Professoren Wideburg und Stolle wiesen als Befürworter der Nostrifikation Amos in Jena unter anderem darauf hin, dieser habe die „commiseration“ verdient, da er „aus einem andern Theile der Welt in seiner zarten Jugend entführet“ und vom „heydenthum zu christl. Religion bekehret“ worden sei ${ }^{80}$. Ein Plädoyer zur Gewährung eines universitären Gnadenbrotes? Fast will es so scheinen. Denn Amo habe keine Eltern und keine Angehörigen, an die er sich wenden könne, wolle aber „nun nicht betteln“, sondern sich auf der Grundlage seiner Fähigkeiten ehrlich ernähren. ${ }^{81}$

So scheint aus dem deportierten versklavten Jungen zunächst und für einige wohl recht glückliche Jahre ein aufstrebender junger Wissenschaftler, bald aber ein mittelloser, auf sich allein gestellter Privatdozent geworden zu sein, der dies dürfte sich für Amo spätestens in Jena abgezeichnet haben - auch nur geringe Chancen auf eine gesicherte Stellung innerhalb der Professorenschaft in Deutschland haben würde. Die eigenhändig niedergeschriebene Vorlesungsankündigung in Jena unterzeichnete der Autor des Tractatus, dessen Beharrlichkeit immer wieder beeindruckt, mit seinem selbstgewählten Namenszusatz „Afer“ und seinem Status als „Magister legens““. 82

Mit dem Regierungsantritt Friedrichs II. von Preußen, der den von seinem Vater verbannten Christian Wolff nach Halle zurückrief, brach für Preußen im Kontext einer nicht allein europäischen, sondern auch weltweit $\mathrm{zu}$ verstehenden Aufklärungsbewegung eine Zeit intensiver geistiger Anstöße an. Allerdings gibt es

79 Ebda., S. 61.

80 Zit. nach ebda., S. 63.

81 Ebda.

82 Abdruck des Faksimile in Brentjes, Burchard: Anton Wilhelm Amo. Der schwarze Philosoph in Halle, S. 66. 
bislang keinerlei Belege dafür, dass Amo noch ein drittes Mal versucht haben könnte, nach Halle zurückzukehren oder etwa an die Viadrina nach Frankfurt an der Oder zu wechseln - Berlin verfügte damals bekanntlich nicht über eine eigene Universität. Die Rückholung Wolffs aus der Verbannung musste auch auf Amo Eindruck gemacht haben. Und so kann man mit guten Gründen spekulieren, dass Blumenbachs Informationen, denen zufolge sich Amo am preußischen Hof aufgehalten haben sollte, zutreffend waren. Der Göttinger Anthropologe und Schädelkundler behauptete, Anton Wilhelm Amo sei als Hofrat ${ }^{83}$ an den Preußischen Hof nach Berlin gegangen. William E. Abraham spekulierte zudem - wie vor ihm bereits Wolfram Suchier ${ }^{84}$-, der Ruf an den Hof nach Berlin und Potsdam könne von dem jungen Friedrich II. ausgegangen sein, der 1740 seinem Vater auf dem preußischen Thron nachfolgte. ${ }^{85}$ All dies scheint überaus plausibel, lässt sich bislang aber nicht belegen. Nur: Die von Amo erträumte Professur wäre mit einer solchen Hofratsstelle nicht verbunden gewesen.

Ein möglicher Aufenthalt Amos in Berlin oder Potsdam könnte in der Tat auch erklären, warum es von ihm in der Folge keine Nachrichten mehr aus den Universitätsstädten Halle, Wittenberg oder Jena gab. ${ }^{86}$ Amo war aus dem akademischen Leben für Jahre verschwunden. Zu den wenigen Lebenszeichen des Philosophen zählt ein später Hinweis, dass Amo noch im Jahr 1747 ein letztes Mal in Jena als dort lebender Bürger genannt worden sein soll. ${ }^{87}$ Dies würde einen zeitweiligen Aufenthalt in Berlin jedoch nicht zwangsläufig ausschließen. Vieles spricht mithin für eine ,Rückkehr` Amos nach Preußen auf der Suche nach einer besseren Stellung bei Hofe oder anderswo. Gewiss aber wäre ihm niemals in den Sinn gekommen, sich den Diensten jener ,Hofmohren“ anzuschließen, die ihren Sklavendienst am preußischen Hofe versahen und die ihre Karikatur im ,Mohren-Rondell` $\mathrm{zu}$ Sanssouci erfuhren. Es ist verlockend, sich in jener Zeit, die in Amos Lebenslauf im Dunklen liegt, den in Preußen ausgebildeten Philosophen in Berlin oder in Sanssouci vorzustellen. Doch wird es weitere Recherchen brauchen, um derlei Vor-

83 Vgl. Blumenbach, Johann Friedrich: Von den Negern. Einige naturhistorische Bemerkungen bey Gelegenheit einer Schweizerreise, S. 9; für wenig glaubhaft hält Blumenbachs Darstellung freilich Mabe, Jacob Emmanuel: Anton Wilhelm Amo interkulturell gelesen, S. 34.

84 Vgl. Suchier, Wolfram: Weiteres über den Mohren Amo. In: Altsachsen. Zeitschrift des Altsachsenbundes für Heimatschutz und Heimatkunde (Holzminden) 1-2 (Januar - Februar 1918), S. 7-9, S. 9. Im Geheimen Staatsarchiv zu Berlin war bislang aber entgegen der Hoffnungen Suchiers keine Aufklärung zu finden.

85 Vgl. Abraham, William E.: Anton Wilhelm Amo, S. 197.

86 Vgl. hierzu ebda., S. 197.

87 Vgl. Brentjes, Burchard: Anton Wilhelm Amo. Der schwarze Philosoph in Halle, S. 66. 
stellungen, die auf den gewöhnlich gut informierten Blumenbach zurückgehen, zu konkretisieren.

Früh schon dürfte Amo erkannt haben, dass sich für ihn die Bedingungen seines Schaffens wohl nur schwerlich wieder verbessern würden. Bereits am 5. Mai des Jahres 1740 schrieb er seinem Studenten und Freund Gottfried Achenwall, dem später berühmt gewordenen Begründer der Statistik in Deutschland, einen Satz des von ihm verehrten Epiktet ins Stammbuch: „Necessitati qui se accomodat, sapit estque rerum Divinarum conscius." ${ }^{\text {88 }}$ Und er unterzeichnete seinen Eintrag einmal mehr mit „Antonius Guilielmus Amo Afer / Philos. et Art. Liberal. Magister Legens“. ${ }^{89}$ Die stoische Gelassenheit, die Amo hier an den Tag legte, mag vor dem Hintergrund der gewiss auch stoischen Prägung seiner Philosophie ${ }^{90}$ verdeutlichen, dass er längst begriffen hatte, wie eng die Grenzen waren, welche ihm das akademische Feld für seine Philosophie, für sein eigenes Philosophieren, gesetzt hatte. Man könnte das Diktum Epiktets, des späten Vertreters der stoischen Philosophie, wie folgt übersetzen: „Wer sich der Notwendigkeit anzupassen weiß, ist weise und sich göttlicher Dinge bewusst.“

Die vierziger Jahre standen für Amo in Deutschland gewiss unter keinem guten Stern. Sein letzter großer Förderer, Johann Peter von Ludewig, verstarb 1743, was ihn sicherlich mancher Hoffnungen beraubte, doch noch einen Weg zu einer sicheren Stellung an eine Universität in Deutschland zu finden. Nach dem Tod von Ludewigs verschlimmerte sich Amos Situation wohl weiter. Im Jahr 1747 wurde gar ein übles Schmähstück über ihn verfasst, das ohne jeden Zweifel protorassistische Angriffe gegen ihn enthielt. Im Zentrum dieses verleumderischen Machwerks stand der gelehrte Mohr Amo, der unsägliche Anträge an eine schöne Brünette macht. Doch das deutsche Mädchen lacht den Freier wegen seiner körperlichen Attribute aus und schickt den so lächerlich Gemachten wieder nach Hause. ${ }^{91}$ Das Schmähstück gibt uns einen Eindruck von jener immer rassistischer werdenden Atmosphäre, in der Anton Wilhelm Amo sich in deutschen Landen mittlerweile bewegen musste. Seine brillante Gelehrsamkeit diente seinen Neidern und Spöttern nur mehr dazu, sich über ihn noch zusätzlich lustig zu machen.

88 Zit. nach Suchier, Wolfram: A.W. Amo, S. 446. Auf einen zweiten Stammbucheintrag vom 3. März 1746 macht aufmerksam Firla, Monika: Ein Jenaer Stammbucheintrag des schwarzen Philosophen Anton Wilhelm Amo aus dem Jahr 1746, S. 16 f. Dort spricht Firla auch von einer von Amo angestrebten Synthese zwischen Epiktet und dem Christentum (ebda., S. 21).

89 Zit. nach Suchier, Wolfram: A.W. Amo, S. 446.

90 Vgl. hierzu Firla, Monika: Ein Jenaer Stammbucheintrag des schwarzen Philosophen Anton Wilhelm Amo aus dem Jahr 1746, S. $7 \mathrm{f}$.

91 Ein Abdruck dieses literarisch stümperhaften Schmähgedichts findet sich unter http:// www.theamoproject.org. 
Es stimmt traurig, dass der Rassist avant la lettre ein Professor war. Denn Autor jener Verunglimpfungen in gereimter Form gegen den „gelehrten Mohren“ war ein ehemaliger Mitstudent Anton Wilhelm Amos, der zeitweilige Professor der deutschen Sprache und Beredsamkeit Johann Ernst Philippi. ${ }^{92}$ Man sollte diesem Machwerk nicht zu viel Gewicht beimessen! Denn ob man daraus auf eine reale Begebenheit der Zurückweisung Amos als Liebhaber in spe schließen darf, ist mehr als fraglich. Wirkliche Belege für die Stichhaltigkeit einer derartigen Begebenheit gibt es nicht. Doch die Existenz dieses Stückes zeugt ohne jeden Zweifel davon, welch wachsenden Anfeindungen sich der Philosoph in Deutschland ausgesetzt sah. Vor allem aber gibt diese unschöne ,Affäre` den Blick frei auf die unverkennbar rassistischen Vorurteile und auch mancherlei Angriffe, denen Anton Wilhelm Amo im Verlaufe seines Lebens in Europa und nicht zuletzt auch während seiner Zeit an deutschen Universitäten ausgesetzt gewesen war. Dass der schwarze deutsche Philosoph und „doctissimus magister legens“ eine Figur des öffentlichen Lebens war, die fraglos enorme Aufmerksamkeit auf sich zog, mögen Philippis Schmähreden sicherlich belegen. Doch als diese Spottverse und Gelegenheitsergüsse erschienen, hatte der seinen Gegnern an Geisteskräften weit überlegene Amo - wie wir noch sehen werden - die Universitätsstadt Jena, Preußen, Deutschland und Europa längst hinter sich gelassen. Und wie die erste Phase seines Lebens abrupt mit einer Reise geendet hatte, so begann die letzte Phase dieses Lebensweges wiederum mit dem Besteigen eines Schiffes. Anton Wilhelm Amo hatte einen Entschluss gefasst. Folgte er aus seiner Sicht dem Diktum Epiktets? In jedem Fall stellte er sich philosophisch einer gewaltigen Herausforderung, die ihn noch einmal weit über die Grenzen seiner Zeit hinaus führen sollte.

Noch hatte Amo seine Reise in eine ihm unbekannte afrikanische Herkunft nicht angetreten. Ein Stammbucheintrag ${ }^{93}$ von Amos Hand am 3. März 1746 belegt wohl zweifelsfrei, dass sich Anton Wilhelm Amo zu diesem Zeitpunkt noch in Deutschland aufhielt. Doch hatte er wohl bereits bei der holländischen

92 Brentjes, Burchard: Anton Wilhelm Amo. Der schwarze Philosoph in Halle, S. 70; nähere Ausführungen hierzu auch bei Abraham, William E.: Anton Wilhelm Amo, S. 197f. Unklar sind die Quellen für die von Johannes Glötzner erzählte Geschichte einer Liebe, die auch mit einem gemeinsamen Kind und damit einem Nachfahren Anton Wilhelm Amos in Deutschland ihren Höhepunkt erreicht: vgl. Glötzner, Johannes: Anton Wilhelm Amo. Ein Philosoph aus Afrika im Deutschland des 18. Jahrhunderts, S. 17. Ist dies nur eine weitere der mittlerweile recht zahlreichen Amo-Legenden? Nicht gänzlich unschuldig an zahlreichen Ausschmückungen könnte die Tatsache sein, dass Johannes Glötzner der Verfasser des wohl ersten Anton Wilhelm Amo gewidmeten Romans ist.

93 Vgl. Firla, Monika: Ein Jenaer Stammbucheintrag des schwarzen Philosophen Anton Wilhelm Amo aus dem Jahr 1746, S. 15-22. 
West-Indischen Kompanie einen Antrag beziehungsweise ein Bittschreiben eingereicht, in dem er um eine kostenlose Passage an die Goldküste ersuchte ${ }^{94}$. Die mächtige Handelsgesellschaft, die ihn einst nach Europa entrissen hatte, sollte ihn wieder an die Küsten Afrikas transportieren. Dies war eine ungeheuer mutige Entscheidung!

Seinem Gesuch wurde stattgegeben. Und so dürfte Amo im Spätjahr 1746 Jacob Emmanuel Mabe gibt allerdings als Abreisedatum von Rotterdam den 20. Dezember 1746 an $^{95}$ - oder spätestens Anfang ${ }^{96}$ des Jahres 1747 Deutschland verlassen haben. Da Amos Schiff bereits im April 1747 die westafrikanische Küste erreichte - Mabe datiert hier wiederum präzise auf den 7. April diesen Jahres ${ }^{97}$-, dürfte er sich unmittelbar nach seinem Verlassen Deutschlands in Holland eingeschifft und Axim im heutigen Ghana wohl problemlos nach sicherer Schifffahrt erreicht haben. ${ }^{98}$ Ein retour au pays natal? Back to Africa? Hatte Amo also die Heimreise angetreten?

Nichts wäre irreführender, als diesem simplistischen Glauben anzuhängen. Die wichtigste Quelle für unser Wissen über die letzten Lebensjahre Amos in Westafrika bildet noch immer der Bericht des Schweizer Reisenden und Schiffsarztes in holländischen Diensten, David Henri Gallandat. Die von ihm im Jahre 1752 aufgezeichneten Notizen erschienen in den Verhandelingen, uitgegeven door het Zeeuwsch Genootschap der Wetenschappen te Vlissingen im Jahr 1782. ${ }^{99}$ Ohne

94 Ebda., S. 25. Vgl. hierzu auch Mabe, Jacob Emmanuel: Anton Wilhelm Amo interkulturell gelesen, S. 26.

95 Ebda.

96 Firla, Monika: Ein Jenaer Stammbucheintrag des schwarzen Philosophen Anton wilhelm Amo aus dem Jahr 1746, S. 25.

97 Vgl. Mabe, Jacob Emmanuel: Anton Wilhelm Amo interkulturell gelesen, S. 26.

98 Näheres zur Schiffsreise Amos und zu seiner „Rückkehr in die Heimat“ vgl. Firla, Monika: Ein Jenaer Stammbucheintrag des schwarzen Philosophen Anton Wilhelm Amo aus dem Jahr 1746, S. 26-31.

99 Zit. nach Brentjes, Burchard: Anton Wilhelm Amo. Der schwarze Philosoph in Halle, S. 68f. Wegen ihrer großen Bedeutung für die Amo-Forschung sei diese Passage auch im Original angeführt: „Terwyl hy op deeze reis te Axim op de Goudkust in Africa was, ging hy den beroemden Heer Anthonius Guilielmus Amo Guinea Afer, Philosophiae Dr. et Artium Liberalium Magister bezoeken. Hy was een Neger, die ruim 30 Jaaren in Europa verkeerd had. Hy was in den Jaare 1707 in Amsterdam, en werd vereerd aan den Hertog van Brunswyk, Anthoni Ulrich, die hem aan zyn zoon Augustus Wilhelmus gaf. Deeze liet hem studeeren te Hall; en in Wittenberg, waar hy in den Jaare 1727 tot Doctor in de Philosophie en Meester in de Vrye konsten Gepromoveerd werd. Eenigen tyd daarna overleed zyn Meester: dit maakte hem zeer droefgeestig, en deed hem bessuiten naar zyn Vaderland te rug te keeren; hy leefde daer toen als een Heremiet, en had den naam van een Gelukzegger te zyn; hy sprak verscheiden taalen, Hebreeuws, Grieks, Latyn, Fransch, Hoog- en Nederduitsch; was zeer kundig in de Astrologie en Astronomie, en een groot 
jeden Zweifel können die dort zusammengetragenen Nachrichten in ihrem Kern nur aus Amos Mund stammen, doch ist die Mündlichkeit dieses Gesprächs von Gallandat in schriftliche Notizen übertragen worden, die ihrerseits Jahrzehnte später in „niederdeutscher“, mithin niederländischer Sprache gedruckt wurden. Wie auch immer wir die einzelnen aufgezählten Biographeme werten und den Wahrheitsgehalt der Verweise auf Vater, Schwester und Bruder Anton Wilhelm Amos beurteilen mögen, so wird doch deutlich, dass sie genügend Spielraum für jeweils unterschiedliche Spekulationen eröffnen.

Die Vorstellung davon, Amo sei wieder ,in sein Vaterland zurückgekehrt', wie man allenthalben lesen kann, ist im Kern kolonialistisch. Denn Amo kehrte keineswegs dorthin zurück, ,wohin er eigentlich hingehörte‘. Denn er gehörte eigentlich nach Deutschland. Anton Wilhelm Amo aber stand vor der vielleicht größten Herausforderung seines wahrlich bewegten Lebens. Denn er reiste in ein Land, in dem er zwar das Licht der Welt erblickt hatte, das er aber nicht kannte, dessen Sprache er nicht sprach, dessen Kultur er nicht kannte und das überdies unter europäischer Kolonialherrschaft stand. Es dürfte zu den großen Widersprüchen und Rätseln seines Lebens zählen, dass er das Ende seiner Existenz - frei oder erzwungen - im Fort von Chama zubrachte und damit in einer Festung, die unter der Herrschaft der West-Indischen Kompanie in das weltweite System des Sklavenhandels eingebunden war.

Mit seiner Reise nach Afrika verschwand der schwarze Philosoph hinter dem Horizont Europas. Die Bekanntheit von Anton Wilhelm Amo Afer beschränkte sich damals keineswegs auf den deutschsprachigen Raum. In seinen berühmten Ausführungen zu De la littérature des nègres aus dem Jahre 1808 wies Abbé Henri Grégoire, ${ }^{100}$ der jakobinische französische Philosoph und Kämpfer gegen Sklaverei und Sklavenhandel, auf nicht weniger als fünf Seiten auf Anton Wilhelm Amo sowie dessen Herkunft aus Guinea und Aufenthalt an den Universitäten von Halle und Wittenberg hin. Wie in Zedlers Grossem vollständigem Universal Lexicon aller Wissenschaften und Künste, mit dem sich mehrere Übereinstimmungen ergeben, wird unter explizitem Rückverweis auf Blumenbachs Ausführungen gleich zu Be-

Wysgeer; zynde toen omtrent 50 Jaaren oud. Zyn Vader en eene Zuster leefden noch, en woonden vier dagreizen landward in; hy had een Broeder, die Slaaf was in de Colonie van Suriname; naderhand is hy van Axim verhuist en gaan woonen in de Fortres der West-Ind. Comp. St. Sebastiaan, te Chama.“ (Verhandelingen uitgegeven door het Zeeuwsch Genootschap der Wetenschappen te Vlissingen. Middelburg: Pieter Gillissen 1782, S. XIX-XX.)

100 Vgl. hierzu den Hinweis in Damis, Christine: Le philosophe connu pour sa peau noire: Anton Wilhelm Amo, S. 119. 
ginn über Amos Untersuchung der rechtlichen Situation der Schwarzen in Europa aufmerksam gemacht. Dabei wurde die Vielsprachigkeit des Philosophen von Anfang an hervorgehoben: „Amo était versé dans l'astronomie et parloit le latin, le grec, l'hébreu, le français, le hollandais et l'allemand.“101 Darüber hinaus griff Grégoire auf den „hommage public“ des Rektors der Universität Wittenberg zurück, der zurecht in Erinnerung gerufen habe „que Térence aussi étoit d'Afrique“. ${ }^{102}$ Zweifellos wusste der französische Abbé sehr wohl, dass sich schon Terenz den Beinamen „Afer“ gegeben hatte: Er stellte Amo in eine Tradition, die bis in die Antike zurückreichte. Auch wenn Abbé Henri Grégoire die Dauer des Aufenthaltes Amos in Europa deutlich unterschätzte, so wird doch die Dimension der geographischen „Rückkehr“ an die westafrikanische Goldküste erwähnt und zugleich in den Kontext einer „tiefen Melancholie“ gerückt, des französischen „mal du siècle“ an der Wende zum 19. Jahrhundert. $^{103}$

Damit wurden Amo zum ersten (aber keineswegs zum letzten) Mal Züge eines romantischen Helden verliehen, der als Fünfzigjähriger schließlich „la vie d'un solitaire“ geführt habe. ${ }^{104}$ Dem von ihm erwähnten Akademiemitglied Gallandat entlehnte Grégoire zugleich die Biographeme, der Vater und eine Schwester Amos hätten damals noch gelebt, und Amos Bruder sei als Sklave nach Surinam verbracht worden. ${ }^{105}$ Damit waren bei Grégoire im Grunde die wesentlichen biographischen Bezugspunkte entwickelt, die noch heute jede Darstellung Amos strukturieren. Abschließend betont der französische „philosophe“, er habe durch seine Recherchen nicht in Erfahrung bringen können, ob Amo andere Werke veröffentlicht habe und wann genau er verstorben sei. Doch es ist hier nicht der Ort, die Rezeption Anton Wilhelm Amos zwischen der Romantik und den Debatten um die Entkolonialisierung Afrikas aufzuarbeiten ${ }^{106}$. Anton Wilhelm Amos Lebenslauf eignete sich hervorragend dafür, die Stationen eines zwar nicht poetischen, aber philosophischen Cahier d'un retour au pays natal ganz im Sinne von Aimé Césaires

101 Grégoire: Henri: De la Littérature des nègres, ou Recherches sur leurs facultés intellectuelles, leurs qualités morales et leur littérature; suivies de Notices sur la vie et les ouvrages des Nègres qui se sont distingués dans les Sciences, les Lettres et les Arts. Paris: chez Maradan Libraire 1808, S. 198.

102 Ebda., S. 199.

103 Vgl. hierzu Ette, Ottmar: Romantik zwischen zwei Welten, S. 177-190.

104 Ebda. Vgl. hier auch den Eintrag in die Nouvelle Biographie Universelle von 1852, auf den erstmals aufmerksam machte Struck, Bernhard: Nochmals A.W. Amo. In: Akademische Rundschau (Leipzig) V (1916-1917), S. 54-56.

105 Grégoire, Henri: De la Littérature des Nègres, S. 201.

106 Ich habe dies im Schlussteil meiner Monographie zu Anton Wilhelm Amo getan. 
großer, in einer ersten Fassung 1939 erschienenen Schöpfung ${ }^{107}$ nicht nur nachzuzeichnen, sondern historisch beispielhaft zu illustrieren.

Wir sollten uns davor hüten, Anton Wilhelm Amo auf seine ,Afrikanität‘ zu reduzieren und gleichsam afrikanisch zu re-territorialisieren: Die wichtigste Zeit seines Lebens hat der Student und Dozent im deutschsprachigen Raum zugebracht. Es wäre parallel zur Re-Afrikanisierung Amos leicht zu belegen, dass die Schriften und wissenschaftlichen Aktivitäten Anton Wilhelm Amo Afers aus der Geschichte der deutschen Philosophie weitestgehend getilgt wurden. Selbst die biographischen Einträge, die noch zu seinen Lebzeiten etwa in Zedlers Universal Lexicon erschienen waren, verschwanden ebenso wie sein Name, ja selbst die Erinnerung an seinen Namen. Sein Ausschluss aus der Geschichte Preußens und Deutschlands war vollständig und radikal.

So kann man dem eigentlichen ,Wiederentdecker‘ Amos für den zumindest deutschsprachigen Raum, dem Bibliothekar Wolfram Suchier, nur zustimmen, wenn er betont, dass die Beschäftigung mit Amo „weitere Bausteinchen zur deutschen Gelehrtengeschichte“108 erbringen werde. Diese Beschäftigung aber sei begründet, sei Amo doch „denkwürdig“ wegen „des Aufsehens, das er zu seiner Zeit in Deutschland erregte, und wegen seiner Schriften für die Universitäts-, Gelehrten- und Kulturgeschichte“. ${ }^{109}$ Die mit Blick auf Afrika vollzogene Inklusion und die hinsichtlich der deutschen und europäischen Wissenschafts-, Geistes- und Philosophiegeschichte sehr weitreichend durchgeführte Exklusion sind komplementär miteinander verwobene Prozesse, die es in ihrer wechselseitigen Stützung aufzubrechen gilt. Denn sie beruhen stets auf simplen Territorialisierungen und exklusiven Zurechnungen wie Ausschlüssen, welche die Komplexität von Leben und Werk Anton Wilhelm Amos in vielen Fällen wissentlich und willentlich zu reduzieren suchen. Wir sollten heute den Versuch unternehmen, Amo als einen aus Afrika stammenden Philosophen und als einen Vertreter der deutschen Frühaufklärung zu denken und damit seine Position als eine Bewegung zwischen zwei Welten zu bestimmen, welche nicht einseitig fixiert werden darf. Denn Anton Wilhelm Amo ist weit mehr als die Addition beider Einschreibungen und Traditionen, sondern vielmehr der kühne und zugleich immer prekäre Versuch, nicht die Addierung, sondern die Bewegung zwischen diesen Traditionssträngen zu sein und explizit als Afrikaner an der deutschen Frühaufklärung teilzuhaben - und zwar ohne sich auf sie allein reduzieren zu lassen.

107 Vgl. Césaire, Aimé: Cahier d'un retour au pays natal. In: Volontés (Paris) 20 (1939).

108 Suchier, Wolfram: Weiteres über den Mohren Amo, S. 7.

109 Ebda. 
Amos Philosophie ist ganz im Sinne seines Tractatus de arte sobrie et accurate philosophandi eine Kunst des Philosophierens, eines Denkens und Schreibens aus der Bewegung zwischen den Welten, einer Philosophie der Aufklärung ohne festen Wohnsitz. Sie lässt sich nicht auf eine einzige Perspektivik, auf eine einzige Zugehörigkeit, auf einen einzigen Standpunkt reduzieren. Die transareale Vektorisierung eines Lebenswissens, das sich als Überlebenswissen gerade auf dem Feld des Denkens erprobte, hat Amos Philosophieren stark bestimmt, insofern die Querung unterschiedlicher Sprachen, verschiedenartiger Kulturen und oft widersprüchlicher Traditionen eine dynamische Polylogik entstehen ließ, die Theologie wie gewiss auch Philosophie in Amos Denken nachdenklich, aber auch nachdrücklich in den Plural setzte. Eben darin liegt die immense Bedeutung seines Denkens heute.

Im Kontext der deutschen Frühaufklärung und aus deren Tradition heraus warf Anton Wilhelm Amo Fragen auf, die (sich) ein (nur) deutscher Philosoph niemals gestellt hätte. Denn über die Rechtsstellung der Schwarzen in Europa nachzudenken, wäre kaum einem deutschen Denker des 18. Jahrhunderts in den Sinn gekommen. Anton Wilhelm Amo aber wusste, wovon er sprach, wenn von Sklaverei an europäischen Fürstenhöfen die Rede war. Er situierte sich in seinem Denken entschlossen in einem Spannungsverhältnis zwischen Afrika und Deutschland, eröffnete einen Raum des Denkens ohne festen Wohnsitz, den erst die letzten Jahrzehnte unserer heutigen Zeit verständlich und nachvollziehbar gemacht haben. Er begründete damit eine Tradition, die es in Deutschland von Beginn an nicht leicht gehabt hat. Heute jedoch können wir diesen Philosophen besser verstehen und nicht auf eine statische, unbewegliche Position festnageln.

Die Vielzahl an Sprachen, derer er sich bediente und die er souverän meisterte, verraten uns etwas von den Deutschland weit übersteigenden Horizonten seines Denkens, verweisen uns auf Amos Weltbewusstsein, das aus jeder Zeile seiner Schriften spricht. Das Lateinische seines Schreibens war keinem Ort, war keinem ,Vaterlande‘ zuzurechnen. Nein, Amo war nicht aus freien Stücken nach Deutschland gekommen, hatte sich nicht nach Abwägung aller Argumente für Deutschland entschieden. Aber er nahm Deutschland als jenen Ort an, von dem aus er zu denken begonnen, den er zu seinem Reflexions-Ort gemacht hatte und dessen Denktraditionen er mit seinem Philosophieren ohne festen Wohnsitz bereichern wollte. Ja, er war als Sklave an einen Fürstenhof gekommen, war nicht mehr als ein Geschenk gewesen, ein Objekt, mit dessen Hilfe man gute Geschäftsbeziehungen bekräftigen wollte. Ein Luxusartikel sozusagen. Doch der Luxusartikel dachte nach und reflektierte über sein eigenes Schicksal wie auch das vieler anderer, die ein rassistisches Kolonialsystem in alle Ecken der Welt verbracht hatte. Er war Opfer einer unmenschlichen Asymmetrie der Macht. Damit war und ist er ein Vorbild für all jene, die ebenfalls nicht freiwillig nach Deutschland kamen, aber 
Deutschland zu ihrem Lebens- und Denk-Raum gemacht haben. Auch hiermit begründete Amo eine Tradition, die wichtig ist für unser Land. Denn das Objekt war zum Subjekt geworden und ließ uns an diesem schwierigen Subjektwerdungsprozess teilhaben.

Vergessen wir nicht: Amo ist Teil einer Geschichte des Rassismus, die hierzulande stattgefunden hat und weiter stattfindet! Wir sollten ihn gleichwohl nicht politisch instrumentalisieren. Der Verweis darauf, dass schwarze Leben zählen, sollte ihn nicht vor einen ideologischen Karren spannen, sondern unseren Blick dafür öffnen, dass sein Leben für uns zählt und in Zukunft immer stärker zählen wird. Anton Wilhelm Amo ist aus unserer Geschichte nicht mehr wegzudenken: Er ist der Fremde, der zu unserem Eigenen geworden ist und unser eigenes FremdSein mit dem Licht der Aufklärung beleuchtet.

Wenn sich Amo ganz bewusst an einer preußischen Universität als Afrikaner einschrieb, so ließ er von Beginn an keinen Zweifel daran, dass er sich im Bewegungsraum zwischen Afrika und Europa situierte. Er wollte nicht verdrängen, dass er als Sklave herbeigeschafft und verschenkt worden war, er wollte nicht geflissentlich übersehen, dass er in Afrika geboren worden war. In seinen Schriften zeigen sich ständige Blickwechsel, welche belegen, dass er seine Philosophie von mehreren Orten zugleich aus entwickelte und dabei in mehreren Logiken zugleich zu denken vermochte. Dieses viellogische, polylogische Denken bildet eine kostbare Tradition in einem Land, das nicht immer auf der Höhe derartiger Denkmöglichkeiten argumentierte. Es handelt sich dabei vielleicht um das Kostbarste überhaupt: die Fähigkeit, in verschiedenen Logiken gleichzeitig denken zu können. Wir dürfen nicht übersehen, dass Amo ein Philosophieren im Zwischenraum und eine Philosophie ohne festen Wohnsitz entfaltete, welche brutal durch den gegen ihn entfachten Rassismus zum Schweigen gebracht wurde.

Das Schweigen jener deutschen Philosophen, die zweifellos von Amos Existenz wussten - war Amo doch zu seiner Zeit (wie wir sahen) eine Berühmtheit, die in der Wikipedia des 18. Jahrhunderts einen langen Eintrag besaß -, war sicherlich (proto-)rassistisch motiviert. Er verfügte über eine internationale Notorietät, welche ein Immanuel Kant in seiner strikten Ablehnung alles Afrikanischen wie viele seiner Kollegen nicht zur Kenntnis nehmen wollte. All dies wiegt schwer und lastet noch immer auf einer gerechten Einschätzung der wunderbaren intellektuellen Fähigkeiten dieses Philosophen. Und was ist mit dem Schweigen der deutschen Philosophen heute? Die Frage stellt sich durchaus, warum es auch heute noch nicht karrierefördernd zu sein scheint, in Deutschland über diesen schwarzen Philosophen eine Monographie zu verfassen. Die Amo'sche Philosophie ist längst Teil unserer Denktradition - auch und gerade weil sie aus unserem Denken wie aus unseren Traditionen ganz bewusst ausgeschlossen wurde. Doch schwarze Leben zählen. 
Amos Leben zeigt auch, dass Toleranz allein zur Gestaltung einer Gemeinschaft und Gesellschaft nach dem Rassismus nicht ausreicht. Denn das Versuchsobjekt Amo wurde zwar rasch zum Vorzeigeobjekt, in dessen Licht man sich sonnen konnte; doch tolerieren heißt, wie schon Goethe wusste, letztendlich dulden; und als es keine Geduld mehr für die Duldung gab, fiel das Vorzeigeobjekt schnell in Ungnade. Es braucht nicht Toleranz, sondern Achtung und Respekt vor jenem, der für manche das Fremde repräsentiert, im Grunde aber nur jenes Fremde in uns ist, das wir doch so dringend benötigen, um uns selbst besser und tiefer zu verstehen.

Wir dürfen ohne Übertreibung festhalten: Amo, der deutsche Aufklärungsphilosoph, ist wieder da! Sein Leben wie sein Denken sollten bewusst als die frühen Anfänge von Traditionen verstanden werden, die über lange Zeit verschüttet waren, den Schichten der Geschichte entstiegen und nun langsam wieder an der Erdoberfläche erscheinen. Anton Wilhelm Amo in Deutschland? Ein Glücksfall für das Denken - nicht nur der Aufklärung. Ihm gebührt ein fester Platz innerhalb einer im deutschsprachigen Raum angesiedelten Geistesgeschichte und Philosophie im Spannungsfeld eines Denkens und Schreibens ohne festen Wohnsitz zwischen Afrika und Europa: einer Aufklärung zwischen den Welten. 